\title{
ON CONFLUENCES OF GENERAL HYPERGEOMETRIC SYSTEMS
}

\author{
HIRONOBU KIMURA AND KYOICHI TAKANO
}

\begin{abstract}
We extend the well-known results about the process of confluence for the Gauss hypergeometric differential equation to the case of general hypergeometric systems. We see that the process of confluence comes from the geometry of the set of regular elements of the Lie algebra of complex general linear group. As a consequence, we give a geometric and group-theoretic view on the process of confluence for classical special functions.
\end{abstract}

\section{INTRODUCTION}

Inspired by the works of Aomoto [1] and Gel'fand et al. [7, 8, 9], we introduced in [16], for any given partition $\lambda$ of $n$, the general hypergeometric functions of type $\lambda$ defined on the space $Z$ of $r \times n$ complex matrices of rank $r$, where $r$ and $n$ are positive integers with $r<n$. They are defined as solutions of the system of partial differential equations on $Z$ called the general hypergeometric system of type $\lambda$. Outside the singular locus of the system, its solution has the integral representation, which is the "Radon transform" of the character of the universal covering group of the maximal abelian subgroup $H_{\lambda}$ of $\mathrm{GL}(n, \mathbb{C})$ (see Subsection 2.2). In the case where the partition of $n$ is $\lambda=(1, \ldots, 1)$, our hypergeometric function coincides with the general hypergeometric function due to Aomoto and Gel'fand ([1, 7]), which is a generalization of Gauss hypergeometric function, whose system of partial differential equations has only regular singularities. In the case where $\lambda=(n)$, the general hypergeometric function has already been defined and studied in [9] and it gives a generalization of the classical Airy function $\operatorname{Ai}(x)$. It is well known [11] that the Airy function has the integral representation

$$
\operatorname{Ai}(x)=\frac{1}{2 \pi i} \int_{\Delta} e^{x t-t^{3} / 3} d t
$$

where the path of integration $\Delta$ starts from $\infty$ in a sector in which integrand is exponentially recessive and goes to $\infty$ in another recessive sector (cf. [15]). This integral can be viewed as a simple example of an oscillatory integral whose phase function $x t-t^{3} / 3$ is a deformation of the simple singularity $-t^{3} / 3$ of $A_{2}$-type (cf. [3]). The differential equation which characterizes $\operatorname{Ai}(x)$ as its solution has only one singular point $x=\infty$ of irregular type, unlike the case of the Gauss hypergeometric differential equation. For the partitions $\lambda \neq(1, \ldots, 1),(n)$, our functions provide generalizations of special functions of one variable such as Kummer's confluent hypergeometric function, the Bessel function and the Hermite-Weber function. In fact, the special functions, Gauss, Kummer,

1991 Mathematics Subject Classification. Primary 33C70; Secondary 33C15.

Key words and phrases. Hypergeometric system, confluence, regular element, stratification. 
Bessel, Hermite-Weber and Airy are obtained as the general hypergeometric functions for $r=2, n=4$ in the cases where the partitions of 4 are $(1,1,1,1),(2,1,1),(2,2),(3,1)$ and (4), respectively. See $[2,4,10,11]$ for the classical special functions mentioned above, and see also $[10,19]$ for a relation with the nonlinear integrable systems such as Painlevé equations and Garnier systems.

For the differential equations of these special functions, a kind of limit process, called the confluence of singularities, is known, and it enables us to obtain one special differential equation from another one as illustrated in the following diagram.

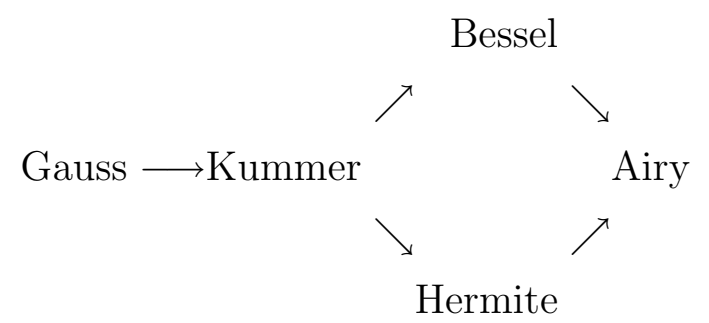

For example, the process of confluence "Gauss $\rightarrow$ Kummer" is described as follows (cf. [2]). The Gauss hypergeometric differential equation is

$$
x(1-x) u^{\prime \prime}+\{c-(a+b+1) x\} u^{\prime}-a b u=0, \quad{ }^{\prime}=d / d x .
$$

This equation, considered in $\mathbb{P}^{1}$, has three regular singular points of $x=0,1, \infty$. For the equation (1.2), we make the change of variable and parameters

$$
x=\varepsilon \xi, \quad b=1 / \varepsilon .
$$

Then the equation for $(\xi, u)$ is

$$
\xi(1-\varepsilon \xi) \frac{d^{2} u}{d \xi^{2}}+\left(c-\varepsilon\left(a+\varepsilon^{-1}+1\right) \xi\right) \frac{d u}{d \xi}-a u=0 .
$$

We see that the coefficients of $d^{2} u / d \xi^{2}, d u / d \xi$ and $u$ depend holomorphically on $\varepsilon$ at $\varepsilon=0$. Taking the limit $\varepsilon \rightarrow 0$ in the equation (1.4), we obtain the Kummer's confluent hypergeometric equation

$$
\xi \frac{d^{2} u}{d \xi^{2}}+(c-\xi) \frac{d u}{d \xi}-a u=0 .
$$

Notice that by the change of variable (1.3) the singular points $x=0,1, \infty$ of (1.2) turn into the singular points $\xi=0,1 / \varepsilon, \infty$ of (1.4), respectively, and that, as $\varepsilon$ tends to 0 , the singular points $\xi=1 / \varepsilon$ and $\xi=\infty$ approach to each other and are amalgamated into the irregular singular point $\xi=\infty$ of (1.5). The name "confluence of singularities" comes from this phenomenon.

It is natural to ask if one can extend the "process of confluence" to the general hypergeometric system and if one can understand the geometrical meaning of the above classically known process for the Gauss differential equation.

So the objective of this paper is summarized as follows: 
- To construct explicitly the limit process by which all the general hypergeometric system for various partition $\lambda \neq(1, \ldots, 1)$ can be obtained from the system of type $\lambda=(1, \ldots, 1)$. We call this limit process the process of confluence.

- To clarify a relation between the geometry of stratification for the set of regular elements of the Lie algebra $\mathfrak{g l}(n ; \mathbb{C})$ and the process of confluence for the general hypergeometric system.

Indeed, our construction will give directly the process of confluence of singular points of the Gauss hypergeometric equation to Kummer's confluent hypergeometric equation $([17])$.

In order to realize our purpose, we need to enlarge slightly the class of systems of hypergeometric type given in [16] so that we can consider the systems associated with centralizers of regular elements of $\mathfrak{g l}(n, \mathbb{C})$, not only the systems associated with the Lie algebra $\mathfrak{h}_{\lambda}$ (see Definition 2.1).

This paper is organized as follows. In Section 2 we give the definition of the general hypergeometric systems on $Z$, each of which is determined by the centralizer $\mathfrak{h}_{b}$ of a regular element $b \in \mathfrak{g l}(n, \mathbb{C})$ and a weight $\alpha$ of $\mathfrak{h}_{b}$. We also recall results on the integral representation of its solutions. In Section 3 we recall results on a stratification in the set $B$ of regular elements of $\mathfrak{g l}(n, \mathbb{C})$ and describe explicitly the relation of adherence among the strata of $B$ and the properties of each stratum. In Section 4, we give key lemmas, which will be proved in the last section. We study in Section 5 the relation of adherence among the centralizers $\mathfrak{h}_{b}(b \in B)$ in an explicit way. This explicit construction yields a process of confluence among the general hypergeometric systems (Theorem 5.3). Thus we see that the process of confluence among our systems is nothing but the explicit realization of the relation of adherence among the strata of regular elements of $\mathfrak{g l}(n, \mathbb{C})$ and among the maximal abelian Lie subalgebras which are defined as the centralizers of regular elements. In Section 6, we shall show that the process of confluence for the general hypergeometric systems also provides the confluence on the level of integrands of the integral representations. In Section 8, we discuss the process of confluence for the special differential equations in (1.1) and for Appell's hypergeometric system $\left(F_{1}\right)$ in detail in the framework of general hypergeometric systems using Theorem 5.3. Parts of the results of this paper have been announced in [17].

We thank the referee for valuable comments for the improvement of this paper. We thank also Professors M. Noumi and T. Sasaki for helpful discussion with them. The first author thanks Professor F. Pham and the members of Université de Nice for their hospitality during his stay in Nice. A part of this paper was written in Nice.

\section{General hypergeometric systems}

In this section, we reformulate general hypergeometric systems for centralizers of regular elements of $\mathfrak{g l}(n, \mathbb{C})$ and their weights.

2.1. Hypergeometric systems. Let $b \in \mathfrak{g l}(n, \mathbb{C})$ be a regular element, namely, the dimension of its centralizer $\{X \in \mathfrak{g l}(n, \mathbb{C}) \mid[b, X]:=b X-X b=0\}$ is equal to $n$, the 
rank of $\mathfrak{g l}(n, \mathbb{C})$. If $b$ has $l$ distinct eigenvalues $b^{(0)}, \ldots, b^{(l-1)}$ of multiplicities $\lambda_{0}, \ldots, \lambda_{l-1}$ with $\lambda_{0} \geq \lambda_{1} \geq \cdots \geq \lambda_{l-1}$ and $\lambda_{0}+\cdots+\lambda_{l-1}=n$, then it is expressed as

$$
b=\left(\operatorname{Ad} g_{b}\right)\left(\left(b^{(0)} I_{\lambda_{0}}+\Lambda_{\lambda_{0}}\right) \oplus \cdots \oplus\left(b^{(l-1)} I_{\lambda_{l-1}}+\Lambda_{\lambda_{l-1}}\right)\right)
$$

for some $g_{b} \in \operatorname{GL}(n, \mathbb{C})$ and its centralizer, denoted by $\mathfrak{h}_{b}$, is given as

$$
\mathfrak{h}_{b}=\left(\operatorname{Ad} g_{b}\right)\left(\mathfrak{j}\left(\lambda_{0}\right) \oplus \cdots \oplus \mathfrak{j}\left(\lambda_{l-1}\right)\right) \text {. }
$$

Here, for any positive integer $m, I_{m}$ denotes the identity matrix of size $m, \Lambda_{m}=\left(\delta_{i+1, j}\right)_{0 \leq i, j<m}$ is the shift matrix of size $m$ and

$$
\mathfrak{j}(m)=\left\{\sum_{0 \leq i<m} x_{i} \Lambda_{m}^{i} \mid x_{i} \in \mathbb{C}\right\} .
$$

We consider the sequence $\lambda:=\left(\lambda_{0}, \ldots, \lambda_{l-1}\right)$ as a partition of $n$ and denote by $\mathbf{Y}_{n}$ the set of partitions of $n$. For $\lambda=\left(\lambda_{0}, \ldots, \lambda_{l-1}\right) \in \mathbf{Y}_{n}$, we call $l$ the length of $\lambda$ and denote it by $\ell(\lambda)$. Let $B$ be the set of regular elements of $\mathfrak{g l}(n, \mathbb{C})$ and $B_{\lambda}$, for $\lambda=\left(\lambda_{0}, \ldots, \lambda_{l-1}\right) \in \mathbf{Y}_{n}$, the subset of $B$ whose element has $l$ distinct eigenvalues of multiplicities $\lambda_{0}, \ldots, \lambda_{l-1}$. Then

$$
B=\bigsqcup_{\lambda \in \mathbf{Y}_{n}} B_{\lambda} \quad \text { (disjoint union) }
$$

If we use the notation

$$
\mathfrak{h}_{\lambda}:=\mathfrak{j}\left(\lambda_{0}\right) \oplus \cdots \oplus \mathfrak{j}\left(\lambda_{l-1}\right)
$$

then we have

$$
\mathfrak{h}_{b}=\left(\operatorname{Ad} g_{b}\right) \mathfrak{h}_{\lambda}
$$

Note that $\mathfrak{h}_{\lambda}=\mathfrak{h}_{b}$ when $g_{b}=I_{n}$.

Now let $r$ and $n$ be positive integers with $r<n$ and $Z$ the set of $r \times n$ complex matrices of rank $r$. We denote by $z=\left(z_{i j}\right)_{0 \leq i<r, 0 \leq j<n}$ the coordinates of $Z$, and by $\partial_{z}=\left(\partial_{i j}\right)$ the matrix whose $(i, j)$ entry is the partial derivation $\partial_{i j}:=\partial / \partial z_{i j}$.

Let $\mathfrak{h}_{b}^{*}$ be the dual space of $\mathfrak{h}_{b}$ and $\langle\cdot, \cdot\rangle$ the canonical bilinear pairing $\mathfrak{h}_{b}^{*} \times \mathfrak{h}_{b} \rightarrow \mathbb{C}$.

Definition 2.1. For a regular element $b$ of $\mathfrak{g l}(n, \mathbb{C})$ and $\alpha \in \mathfrak{h}_{b}^{*}$ satisfying the condition

$$
\left\langle\alpha, I_{n}\right\rangle=-r
$$

the following system of partial differential equations

$$
\left\{\begin{aligned}
L_{X} u & :=\left\{\operatorname{Tr}\left(z X^{t} \partial_{z}\right)-\langle\alpha, X\rangle\right\} u=0, \quad X \in \mathfrak{h}_{b}, \\
M_{Y} u & :=\operatorname{Tr}\left(Y z^{t} \partial_{z}+Y\right) u=0, \quad Y \in \mathfrak{g l}(r, \mathbb{C}), \\
\square_{i i^{\prime}, j j^{\prime}} u & :=\left\{\partial_{i j} \partial_{i^{\prime} j^{\prime}}-\partial_{i j^{\prime}} \partial_{i^{\prime} j}\right\} u=0, \quad 0 \leq i, i^{\prime}<r, \quad 0 \leq j, j^{\prime}<n
\end{aligned}\right.
$$

is called the general hypergeometric system associated with $\left(\alpha, \mathfrak{h}_{b}\right)$ (GHG system for short). Let $\mathbb{C}\left[z_{i j}, \partial_{i j}(0 \leq i<r, 0 \leq j<n)\right]$ be the Weyl algebra and $\mathcal{I}\left(\alpha ; \mathfrak{h}_{b}\right)$ its left ideal generated by $L_{X}\left(X \in \mathfrak{h}_{b}\right), M_{Y}(Y \in \mathfrak{g l}(r, \mathbb{C}))$ and $\square_{i i^{\prime}, j j^{\prime}}\left(0 \leq i, i^{\prime}<r, 0 \leq j, j^{\prime}<n\right)$. We often identify the system with the left ideal $\mathcal{I}\left(\alpha ; \mathfrak{h}_{b}\right)$. 
Remark 2.1. (2.4) is a compatibility condition of the system. Indeed, if $X=I_{n}$ and $Y=I_{r}$ in (2.5), the equations $L_{X} u=0$ and $M_{Y} u=0$ coincide with each other and then $\left\langle\alpha, I_{n}\right\rangle=-\operatorname{Tr}\left(I_{r}\right)=-r$.

Remark 2.2. The equations $L_{X} u=0$ and $M_{Y} u=0$ imply that a solution $u(z)$ of $(2.5)$ satisfies

$$
\begin{aligned}
u(z \exp (t X)) & =u(z) \chi_{b}(\alpha ; \exp (t X)), \quad X \in \mathfrak{h}_{b}, \\
u(g z) & =\operatorname{det}\left(g^{-1}\right) u(z), \quad g \in \operatorname{GL}(r, \mathbb{C}),
\end{aligned}
$$

where $\chi_{b}(\alpha ; \cdot)$ is that defined in (2.8). The third equations $\square_{i i^{\prime}, j j^{\prime}} u=0$ of $(2.5)$ are those of ultrahyperbolic type used in characterizing the range of Radon transform, see [6], [12] and [13].

Remark 2.3. The system (2.5) is a holonomic system on an Zariski open set of $Z$. The dimension of the solution space at a generic point of $Z$ is conjectured to be $\left(\begin{array}{l}n-2 \\ r-1\end{array}\right)$. This is true for the system with $b$ of type $\lambda=(1, \ldots, 1),(n)$ and any $r \geq 2$. It is also true in the cases $r=2$ with $b$ of any type $\lambda$.

2.2. Integral representation. The GHG system $\mathcal{I}\left(\alpha ; \mathfrak{h}_{b}\right)$ for $b \in B$ and $\alpha \in \mathfrak{h}_{b}^{*}$ has solutions given by definite integrals whose integrand is expressed in terms of a character $\chi_{b}(\alpha ; \cdot)$ of the simply connected Lie group $\tilde{H}_{b}$ of the Lie algebra $\mathfrak{h}_{b}$, where the character is determined by the following commutative diagram:

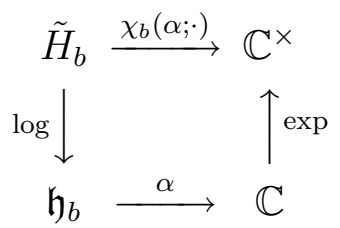

We give here explicit expressions of the character and the integrand.

For any $b \in B_{\lambda}$ given by (2.1) for some $g_{b} \in \mathrm{GL}(n, \mathbb{C})$, we define $H_{b}$, a maximal abelian subgroup of $\mathrm{GL}(n, \mathbb{C})$, by

$$
H_{b}=\left(\operatorname{Ad} g_{b}\right)\left(J\left(\lambda_{0}\right) \times \cdots \times J\left(\lambda_{l-1}\right)\right),
$$

where $J(m)$, for any positive integer $m$, is the matrix group

$$
J(m)=\left\{h=\sum_{0 \leq i<m} h_{i} \Lambda_{m}^{i} \mid h_{i} \in \mathbb{C}, h_{0} \neq 0\right\} \subset \mathrm{GL}(m, \mathbb{C}),
$$

called the Jordan group of size $m$. Then $H_{b}$ is a Lie group of $\mathfrak{h}_{b}$ and its universal covering group coincides with $\tilde{H}_{b}$. Corresponding to (2.3), we use the notation

$$
H_{\lambda}:=J\left(\lambda_{0}\right) \times \cdots \times J\left(\lambda_{l-1}\right) .
$$

Then

$$
H_{b}=\left(\operatorname{Ad} g_{b}\right) H_{\lambda}
$$

and $H_{b}=H_{\lambda}$ when $g_{b}=I_{n}$. 
Let us obtain an explicit expression of the character $\chi_{b}(\alpha ; \cdot)$. Suppose first $g_{b}=I_{n}$. In this case, we also denote $\chi_{b}(\alpha ; \cdot)$ by $\chi_{\lambda}(\alpha ; \cdot)$. Since $\tilde{H}_{\lambda}$ is a direct product of $\tilde{J}\left(\lambda_{k}\right), 0 \leq$ $k<\ell(\lambda)$, we suppose further $\lambda=(n)$.

Here we introduce functions $\theta_{i}(z), i \geq 0$, of infinitely many variables $z=\left(z_{0}, z_{1}, \ldots\right)$ with $z_{i} \in \mathbb{C}, i \geq 0, z_{0} \neq 0$, defined by

$$
\sum_{i \geq 0} \theta_{i}(z) t^{i}=\log \left(\sum_{i \geq 0} z_{i} t^{i}\right)=\log \left(z_{0}\right)+\log \left(1+\sum_{i \geq 1}\left(z_{i} / z_{0}\right) t^{i}\right)
$$

as formal power series of $t$, where $\log 1=0$. Notice that $\theta_{0}(z)=\log z_{0}$ and each $\theta_{i}(z), i \geq$ 1 , is a weighted homogeneous polynomial of $z_{1} / z_{0}, \ldots, z_{i} / z_{0}$ of weight $i$, where the weight of $z_{k} / z_{0}$ is defined to be $k$.

Now let $h=\sum_{0 \leq i<n} h_{i} \Lambda_{n}^{i} \in \tilde{J}(n)$ and $\alpha \in \mathfrak{h}_{(n)}^{*}$. Then we have

$$
\begin{aligned}
\log h & =\log \left(\sum_{0 \leq i<n} h_{i} \Lambda_{n}^{i}\right)=\left(\log h_{0}\right) I_{n}+\log \left(I_{n}+\sum_{1 \leq i<n}\left(h_{i} / h_{0}\right) \Lambda_{n}^{i}\right) \\
& =\left(\log h_{0}\right) I_{n}+\sum_{1 \leq i<n} \theta_{i}\left(h_{0}, \ldots, h_{i}\right) \Lambda_{n}^{i}
\end{aligned}
$$

because $\Lambda_{n}^{i}=0$ for any $i \geq n$. Hence, setting

$$
\alpha_{i}:=\left\langle\alpha, \Lambda_{n}^{i}\right\rangle, \quad 0 \leq i<n,
$$

we obtain

$$
\begin{aligned}
\chi_{(n)}(\alpha ; h) & =\exp (\langle\alpha, \log h\rangle)=\exp \left(\left\langle\alpha, \sum_{0 \leq i<n} \theta_{i}\left(h_{0}, \ldots, h_{i}\right) \Lambda_{n}^{i}\right\rangle\right) \\
& =\exp \left(\sum_{0 \leq i<n} \alpha_{i} \theta_{i}\left(h_{0}, \ldots, h_{i}\right)\right)=h_{0}^{\alpha_{0}} \exp \left(\sum_{1 \leq i<n} \alpha_{i} \theta_{i}\left(h_{0}, \ldots, h_{i}\right)\right) .
\end{aligned}
$$

Therefore the character of $\tilde{H}_{\lambda}$ for any $\lambda=\left(\lambda_{0}, \ldots, \lambda_{l-1}\right) \in \mathbf{Y}_{n}$ and $\alpha \in \mathfrak{h}_{\lambda}^{*}$ is given as

$$
\chi_{\lambda}(\alpha ; h)=\prod_{0 \leq k<l} \chi_{\left(\lambda_{k}\right)}\left(\alpha^{(k)} ; h^{(k)}\right), \quad h \in \tilde{H}_{\lambda},
$$

where $\alpha^{(k)}:=\left.\alpha\right|_{\mathfrak{j}\left(\lambda_{k}\right)}$ and $h^{(k)} \in \tilde{J}\left(\lambda_{k}\right)$ is the $k$-th component of $h$.

For a general $b \in B_{\lambda}$ where $g_{b} \neq I_{n}$, we have since $\mathfrak{h}_{b}=\left(\operatorname{Ad} g_{b}\right) \mathfrak{h}_{\lambda}$ and $H_{b}=\left(\operatorname{Ad} g_{b}\right) H_{\lambda}$,

$$
\chi_{b}(\alpha ; h)=\chi_{\lambda}\left(\left(\operatorname{Ad} g_{b}\right)^{*} \alpha ;\left(\operatorname{Ad} g_{b}\right)^{-1} h\right), \quad h \in \tilde{H}_{b},
$$

where $\left(\operatorname{Ad} g_{b}\right)^{*}: \mathfrak{h}_{b}^{*} \rightarrow \mathfrak{h}_{\lambda}^{*}$ is the dual of the isomorphism Ad $g_{b}: \mathfrak{h}_{\lambda} \rightarrow \mathfrak{h}_{b}$.

In order to give an integrand of integral representations for solutions of the GHG system $\mathcal{I}\left(\alpha ; \mathfrak{h}_{b}\right)\left(b \in B_{\lambda}\right)$, we introduce an injective mapping $\iota_{b}$ from $H_{b}$ to the space of $n$ dimensional row vectors: $\iota_{b}: H_{b} \rightarrow \mathbb{C}^{n}$. In the case of $g_{b}=I_{n}, \iota_{\lambda}:=\iota_{b}$ is defined by

$$
\iota_{\lambda}(h)=\left(h_{0}^{(0)}, \ldots, h_{\lambda_{0}-1}^{(0)}, \ldots, h_{0}^{(l-1)}, \ldots, h_{\lambda_{l-1}-1}^{(l-1)}\right)
$$

for $h=\oplus_{0 \leq k<l} \sum_{0 \leq i<\lambda_{k}} h_{i}^{(k)} \Lambda_{\lambda_{k}}^{i} \in H_{\lambda}$. For a general $b \in B_{\lambda}$ where $g_{b} \neq I_{n}$, it is defined by

$$
\iota_{b}=R_{g_{b}}^{-1} \circ \iota_{\lambda} \circ\left(\operatorname{Ad} g_{b}\right)^{-1},
$$


where $R_{g_{b}}$ denotes the right multiplication operator by $g_{b}$. Notice that $\iota_{b}\left(H_{b}\right)=\left(\prod_{0 \leq k<l}\left(\mathbb{C}^{\times} \times\right.\right.$ $\left.\left.\mathbb{C}^{\lambda_{k}-1}\right)\right) g_{b}^{-1}$. This mapping can be lifted to a biholomorphic mapping from $\tilde{H}_{b}$ to the universal covering space of $\left(\prod_{0 \leq k<l}\left(\mathbb{C}^{\times} \times \mathbb{C}^{\lambda_{k}-1}\right)\right) g_{b}^{-1}$, which is also denoted by the same symbol $\iota_{b}$. In the same way, we define a bijective mapping denoted also by $\iota_{b}$ :

$$
\iota_{b}: \mathfrak{h}_{b} \rightarrow \mathbb{C}^{n}=\left(\bigoplus_{0 \leq k<l} \mathbb{C}^{\lambda_{k}}\right) g_{b}^{-1}
$$

Notice that for any row vector $s=\left(s_{0}, \ldots, s_{n-1}\right) \in \mathbb{C}^{n}$ and $h \in \tilde{H}_{b}$, it holds that

$$
\iota_{b}^{-1}(s h)=\iota_{b}^{-1}(s) h
$$

From this and the fact that $\chi_{b}(\alpha ; \cdot)$ is a character, we obtain

$$
\chi_{b}\left(\alpha ; \iota_{b}^{-1}(t z h)\right)=\chi_{b}\left(\alpha ; \iota_{b}^{-1}(t z)\right) \cdot \chi_{b}(\alpha ; h), \quad h \in \tilde{H}_{b} .
$$

Let $\tau$ be the $(r-1)$-form in $r$ dimensional complex affine space defined by

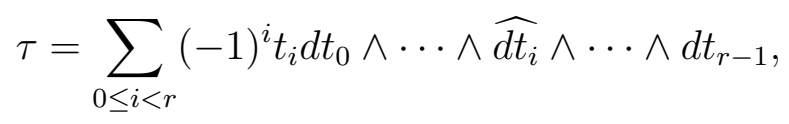

then the $(r-1)$-form $\chi_{b}\left(\alpha ; \iota_{b}^{-1}(t z)\right) \cdot \tau$ is invariant under the homothety $t \mapsto c t\left(c \in \mathbb{C}^{\times}\right)$ by virtue of (2.4), and hence it defines a multivalued complex analytic $(r-1)$-form on an open submanifold of $r-1$ dimensional complex projective space. This $\chi_{b}\left(\alpha ; \iota_{b}^{-1}(t z)\right) \cdot \tau$ is the integrand of integral representations for the system $\mathcal{I}\left(\alpha ; \mathfrak{h}_{b}\right)$, namely integrals

$$
u(z)=\Phi_{b}(\alpha ; z):=\int_{\Delta(z)} \chi_{b}\left(\alpha ; \iota_{b}^{-1}(t z)\right) \cdot \tau
$$

for various twisted cycles $\Delta(z)$ give solutions of the system $\mathcal{I}\left(\alpha ; \mathfrak{h}_{b}\right)$.

\section{Stratification of Regular elements and confluence of GHG systems}

In the following part of this paper, we study confluence process of GHG systems and integral representations of solutions of the systems. In this section, we give an outline.

3.1. Stratification of regular elements. We first define a relation in partitions of $n$.

Definition 3.1. We say that $\mu \in \mathbf{Y}_{n}$ is adjacent to $\lambda \in \mathbf{Y}_{n}$ and write as $\lambda \rightarrow \mu$ if

(1) $\ell(\mu)=\ell(\lambda)-1$, where $\ell(\cdot)$ denotes the length of a partition,

(2) there exist $0 \leq j<\ell(\mu), 0 \leq j_{2}<j_{1}<\ell(\lambda)$ with $\mu_{j}=\lambda_{j_{1}}+\lambda_{j_{2}}$ such that $\left\{\mu_{k}\right\}_{0 \leq k<\ell(\mu), k \neq j}=\left\{\lambda_{k}\right\}_{0 \leq k<\ell(\lambda), k \neq j_{1}, j_{2}}$ as set. 
For example, the adjacent relations among the elements of $\mathbf{Y}_{4}$ are given by

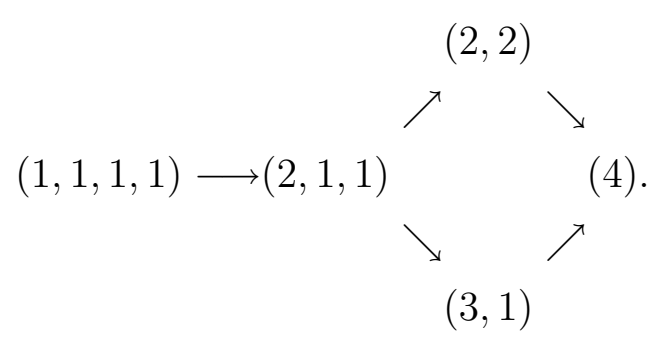

Compare the diagram with that of classical hypergeometric and confluent hypergeometric equations in Section 1.

Now recall $B$ and $B_{\lambda}$ and the decomposition (2.2) of $B$. We notice a well-known fact that (2.2) defines a stratification of $B$ in the sense that each $B_{\lambda}$ is a complex manifold of dimension $n^{2}-n+\ell(\lambda)$ and

$$
\bar{B}_{\lambda}=\bigsqcup_{\mu \leq \lambda} B_{\mu}
$$

where $\overline{B_{\lambda}}$ is the closure of $B_{\lambda}$ in $B$ with respect to the usual topology of $B$ and $\mu \leq \lambda$ means that there is a sequence $\lambda^{\prime}, \lambda^{\prime \prime}, \ldots$ of partitions of $n$ with $\lambda \rightarrow \lambda^{\prime} \rightarrow \lambda^{\prime \prime} \rightarrow \cdots \rightarrow \mu$.

3.2. Outline of confluence process of GHG systems. Let $\mu \in \mathbf{Y}_{n}$ be adjacent to $\lambda \in \mathbf{Y}_{n}$. Then, for any $b \in B_{\mu}$, (3.1) says that there exists a sequence of points in $B_{\lambda}$ which converges to $b$.

We will realize this limit process by constructing a family of mappings $\sigma_{\varepsilon}(\varepsilon \neq 0)$ from $B_{\mu}$ to $B_{\lambda}$ so that $\sigma_{\varepsilon}(b)$ is holomorphic in $\varepsilon$ and $\lim _{\varepsilon \rightarrow 0} \sigma_{\varepsilon}(b)=b$ for any $b \in B_{\mu}$. Notice that, for fixed $b \in B_{\mu}, \varepsilon \mapsto \sigma_{\varepsilon}(b)$ defines a complex analytic curve in $B_{\lambda}$ which tends to $b$ as $\varepsilon \rightarrow 0$. We will next construct a Lie algebra isomorphism $\Psi_{\varepsilon}$ from $\mathfrak{h}_{b}$ to $\mathfrak{h}_{\sigma_{\varepsilon}(b)}$ for $b \in B_{\mu}$. Let $\Psi_{\varepsilon}^{*}$ be the dual isomorphism of $\Psi_{\varepsilon}$ and consider GHG system $\mathcal{I}\left(\left(\Psi_{\varepsilon}^{*}\right)^{-1}(\alpha) ; \mathfrak{h}_{\sigma_{\varepsilon}(b)}\right)$ of type $\lambda$ for any $\alpha \in \mathfrak{h}_{b}^{*}$ with $\left\langle\alpha, I_{n}\right\rangle=-r$. It will be proved that the system $\mathcal{I}\left(\left(\Psi_{\varepsilon}^{*}\right)^{-1}(\alpha) ; \mathfrak{h}_{\sigma_{\varepsilon}(b)}\right)$ converges to the system $\mathcal{I}\left(\alpha ; \mathfrak{h}_{b}\right)$ and integrands of integral representations of solutions of the system $\mathcal{I}\left(\left(\Psi_{\varepsilon}^{*}\right)^{-1}(\alpha) ; \mathfrak{h}_{\sigma_{\varepsilon}(b)}\right)$ converge to those of the system $\mathcal{I}\left(\alpha ; \mathfrak{h}_{b}\right)$ as $\varepsilon \rightarrow 0$. We note that the process reduces to the classical one in each case of $r=2, n=4$.

3.3. A fibration structure of each stratum of $B$. In this subsection, we give a fibration of each stratum of $B$ in order to understand more clearly the mapping $\sigma_{\varepsilon}: B_{\mu} \rightarrow B_{\lambda}$, which will be given in Section 5 .

Let $Y$ be a copy of $\mathbb{C}^{n}$ and $F: B \rightarrow Y$ a mapping which sends $b \in B$ to $F(b)=$ $\left(F_{1}(b), \ldots, F_{n}(b)\right) \in Y$, where

$$
\operatorname{det}(s I-b)=s^{n}-F_{1}(b) s^{n-1}+\cdots+(-1)^{n} F_{n}(b) .
$$

For $\lambda=\left(\lambda_{0}, \ldots, \lambda_{\ell(\lambda)-1}\right) \in \mathbf{Y}_{n}$, set $F_{\lambda}:=\left.F\right|_{B_{\lambda}}$ and

$$
Y_{\lambda}:=F\left(B_{\lambda}\right) \text {. }
$$


We can verify that $Y_{\lambda}$ is an $l$ dimensional complex submanifold of $Y$ and hence the decomposition $Y=\sqcup_{\lambda} Y_{\lambda}$ gives a stratification of $Y$. For $y \in Y_{\lambda}$, let $a^{(0)}(y), \ldots, a^{(\ell(\lambda)-1)}(y)$ be the distinct roots of

$$
t^{n}-y_{1} t^{n-1}+y_{2} t^{n-2}+\cdots+(-1)^{n} y_{n}=0
$$

of multiplicities $\lambda_{0}, \ldots, \lambda_{\ell(\lambda)-1}$ and set

$$
s_{\lambda}(y):=\bigoplus_{0 \leq k<\ell(\lambda)}\left(a^{(k)}(y) I_{\lambda_{k}}+\Lambda_{\lambda_{k}}\right) .
$$

Then $s_{\lambda}(y)$ is in $F_{\lambda}^{-1}(y)$ and the fiber $F_{\lambda}^{-1}(y)$ is the $G$-orbit $O\left(s_{\lambda}(y)\right)$ of $s_{\lambda}(y)$ with respect to the adjoint action of $G:=\operatorname{GL}(n, \mathbb{C})$ on $\mathfrak{g l}(n, \mathbb{C})$ and hence

$$
F_{\lambda}^{-1}(y)=O\left(s_{\lambda}(y)\right) \simeq G / H_{\lambda} .
$$

It is easy to see that the mapping $F_{\lambda}:=\left.F\right|_{B_{\lambda}}: B_{\lambda} \rightarrow Y_{\lambda}$ defines a locally trivial complex analytic fibration and we can take a holomorphic local section of the form (3.2).

\section{KeY LeMmMaS}

In this section, we give lemmas which will play an essential role in constructing the mapping $\sigma_{\varepsilon}: B_{\mu} \rightarrow B_{\lambda}$ and the Lie algebra isomorphism $\Psi_{\varepsilon}: \mathfrak{h}_{b} \rightarrow \mathfrak{h}_{\sigma_{\varepsilon}(b)}$ for $b \in B_{\mu}$.

We first consider a simple case. Let $p$ and $q$ be positive integers. We introduce a matrix $g(\varepsilon) \in \mathrm{GL}(p+q, \mathbb{C})$ depending holomorphically on $\varepsilon \in \mathbb{C}^{\times}$given by

$$
g(\varepsilon)=\left(\begin{array}{cc}
I_{p} & G_{12}(\varepsilon) \\
0 & G_{22}(\varepsilon)
\end{array}\right)
$$

where the $p \times q$ matrix $G_{12}=G_{12}(\varepsilon)$ and $q \times q$ matrix $G_{22}=G_{22}(\varepsilon)$ are defined by

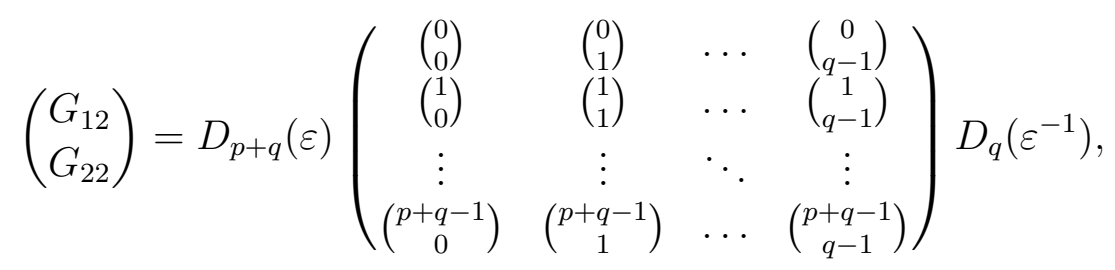

$D_{m}(\varepsilon)$ (for any positive integer $m$ ) denoting $\operatorname{diag}\left(1, \varepsilon, \varepsilon^{2}, \ldots, \varepsilon^{m-1}\right)$ and $\left(\begin{array}{l}i \\ j\end{array}\right)$ denoting the binomial coefficient which is equal to 0 if $i<j$ by the usual convention. We remark that $\operatorname{det} g(\varepsilon)=\varepsilon^{p q}$ and then $g(\varepsilon)$ is nonsingular if $\varepsilon \neq 0$.

Now, for any $X=\sum_{0 \leq i<p+q} x_{i} \Lambda_{p+q}^{i} \in \mathfrak{h}_{(p+q)}$, we define $X(\varepsilon) \in(\operatorname{Ad} g(\varepsilon)) \mathfrak{h}_{(p, q)}$ as follows:

$$
\begin{aligned}
\left(y_{0}(\varepsilon), \ldots, y_{p+q-1}(\varepsilon)\right) & :=\left(x_{0}, \ldots, x_{p+q-1}\right) g(\varepsilon), \\
Y(\varepsilon) & :=\left(\sum_{0 \leq i<p} y_{i}(\varepsilon) \Lambda_{p}^{i}\right) \oplus\left(\sum_{p \leq i<p+q} y_{i}(\varepsilon) \Lambda_{q}^{i-p}\right) \in \mathfrak{h}_{(p, q)}, \\
X(\varepsilon) & :=(\operatorname{Ad} g(\varepsilon)) Y(\varepsilon) .
\end{aligned}
$$

By using the notation introduced in Section 2, we can express it as

$$
X(\varepsilon)=\left((\operatorname{Ad} g(\varepsilon)) \circ \iota_{(p, q)}^{-1} \circ R_{g(\varepsilon)} \circ \iota_{(p+q)}\right)(X) .
$$

Then we have 
Lemma 4.1 (Key lemma 1). For any $X \in \mathfrak{h}_{(p+q)}, X(\varepsilon) \in(\operatorname{Ad} g(\varepsilon)) \mathfrak{h}_{(p, q)}$ is holomorphic in $\varepsilon$ in a neighborhood of $\varepsilon=0$ and

$$
\lim _{\varepsilon \rightarrow 0} X(\varepsilon)=X
$$

The proof of this lemma will be given in Section 7 .

We next give a lemma of more general form, which is an immediate consequence of Lemma 4.1. Let $\mu \in \mathbf{Y}_{n}$ is adjacent to $\lambda \in \mathbf{Y}_{n}$ with $\mu_{j}=\lambda_{j_{1}}+\lambda_{j_{2}}$ for some $0 \leq j<$ $\ell(\mu), 0 \leq j_{2}<j_{1}<\ell(\lambda)$. Then we have a permutation $\rho$ of the set $\{0,1, \ldots, l-1\}$ $(l:=\ell(\lambda))$ defined by

$$
\left(\lambda_{\rho(0)}, \ldots, \lambda_{\rho(l-1)}\right)=\left(\mu_{0}, \ldots, \mu_{j-1}, \lambda_{j_{1}}, \lambda_{j_{2}}, \mu_{j+1}, \ldots, \mu_{l-2}\right) .
$$

Let $g_{\rho} \in \mathrm{GL}(n, \mathbb{C})$ be a permutation matrix determined by

$$
\left(s^{(\rho(0))}, \ldots, s^{(\rho(l-1))}\right) g_{\rho}=\left(s^{(0)}, \ldots, s^{(l-1)}\right)
$$

for any row vector $\left(s^{(0)}, \ldots, s^{(l-1)}\right) \in \mathbb{C}^{n}$, where $s^{(k)}=\left(s_{0}^{(k)}, \ldots, s_{\lambda_{k}-1}^{(k)}\right) \in \mathbb{C}^{\lambda_{k}}, 0 \leq k<l$.

We define a matrix $g_{\lambda \rightarrow \mu}(\varepsilon) \in \mathrm{GL}(n, \mathbb{C})$ by

$$
g_{\lambda \rightarrow \mu}(\varepsilon)=\left(I_{\mu_{0}+\cdots+\mu_{j-1}} \oplus g^{(j)}(\varepsilon) \oplus I_{\mu_{j+1}+\cdots+\mu_{l-2}}\right) g_{\rho},
$$

where $g^{(j)}(\varepsilon) \in \operatorname{GL}\left(\mu_{j} ; \mathbb{C}\right)$ is the matrix determined by (4.1) and (4.2) with $p=\lambda_{j_{1}}$ and $q=\lambda_{j_{2}}$. For $X \in \mathfrak{h}_{\mu}$, we define $X(\varepsilon)$ by

$$
X(\varepsilon)=\left(\left(\operatorname{Ad} g_{\lambda \rightarrow \mu}(\varepsilon)\right) \circ \iota_{\lambda}^{-1} \circ R_{g_{\lambda \rightarrow \mu}(\varepsilon)} \circ \iota_{\mu}\right)(X) .
$$

Then we have

Lemma 4.2 (Key lemma 2). For any $X \in \mathfrak{h}_{\mu}, X(\varepsilon)$ is holomorphic in $\varepsilon$ in a neighborhood of $\varepsilon=0$ and satisfies

$$
\lim _{\varepsilon \rightarrow 0} X(\varepsilon)=X
$$

Proof. Suppose $X=\oplus_{0 \leq k<l-1} X^{(k)}$ with $X^{(k)} \in \mathfrak{j}\left(\mu_{k}\right)$. We can verify that

$$
X(\varepsilon)=\oplus_{0 \leq k \leq j-1} X^{(k)} \oplus\left(\left(\operatorname{Ad} g^{(j)}(\varepsilon)\right) \circ \iota_{\left(\lambda_{j_{1}}, \lambda_{j_{2}}\right)}^{-1} \circ R_{g^{(j)}(\varepsilon)} \circ \iota_{\left(\mu_{j}\right)}\right)\left(X^{(j)}\right) \oplus_{j+1 \leq k<l-1} X^{(k)} .
$$

Then the lemma follows from Lemma 4.1.

\section{Confluence of GHG systems}

\subsection{Convergence of regular elements and Lie algebras.}

Theorem 5.1 (Convergence of regular elements). Suppose that $\lambda \rightarrow \mu, \lambda, \mu \in \mathbf{Y}_{n}$, namely, $\mu$ is adjacent to $\lambda$. Given $b \in B_{\mu}$ with $b \in\left(\operatorname{Ad} g_{b}\right) \mathfrak{h}_{\mu}$, let

$$
\sigma_{\varepsilon}(b):=\left(\left(\operatorname{Ad} g_{b} g_{\lambda \rightarrow \mu}(\varepsilon)\right) \circ \iota_{\lambda}^{-1} \circ R_{g_{\lambda \rightarrow \mu}(\varepsilon)} \circ \iota_{\mu} \circ\left(\operatorname{Ad} g_{b}\right)^{-1}\right)(b)
$$

then $\sigma_{\varepsilon}(b)$ is an element of $B_{\lambda} \cap\left(\operatorname{Ad} g_{b} g_{\lambda \rightarrow \mu}(\varepsilon)\right) \mathfrak{h}_{\lambda}$ for any fixed $\varepsilon$ with $0<|\varepsilon|<<1$, holomorphic in $\varepsilon$ in a neighborhood of $\varepsilon=0$ and satisfies

$$
\lim _{\varepsilon \rightarrow 0} \sigma_{\varepsilon}(b)=b \text {. }
$$


Proof. Let $\mu_{j}=\lambda_{j_{1}}+\lambda_{j_{2}}$ for $0 \leq j<\ell(\mu), 0 \leq j_{2}<j_{1}<\ell(\lambda)$, and let $b^{(0)}, \ldots, b^{(\ell(\mu)-1)}$ be $\ell(\mu)$ distinct eigenvalues of $b$ of multiplicities $\mu_{0}, \ldots, \mu_{\ell(\mu)-1}$. Then we have

$$
c:=\left(\operatorname{Ad} g_{b}\right)^{-1}(b)=\oplus_{0 \leq k<\ell(\mu)}\left(b^{(k)} I_{\mu_{k}}+\Lambda_{\mu_{k}}\right) .
$$

Set $l=\ell(\lambda)$ and

$$
\left(b_{0}^{(0)}(\varepsilon), \ldots, b_{\lambda_{0}-1}^{(0)}(\varepsilon), \ldots, b_{0}^{(l-1)}(\varepsilon), \ldots, b_{\lambda_{l-1}-1}^{(l-1)}(\varepsilon)\right):=\left(R_{g_{\lambda \rightarrow \mu}(\varepsilon)} \circ \iota_{\mu}\right)(c) .
$$

Then we see that

$$
\begin{array}{llrl}
b_{0}^{(k)}(\varepsilon) & =b^{(k)}, & b_{1}^{(k)}(\varepsilon) & =1, \quad b_{i}^{(k)}(\varepsilon)=0,2 \leq i<\lambda_{k}, 0 \leq k<l, k \neq j_{1}, j_{2}, \\
b_{0}^{\left(j_{1}\right)}(\varepsilon)=b^{(j)}, & b_{1}^{\left(j_{1}\right)}(\varepsilon)=1, & b_{i}^{\left(j_{1}\right)}(\varepsilon)=0,2 \leq i<\lambda_{j_{1}}, \\
b_{0}^{\left(j_{2}\right)}(\varepsilon)=b^{(j)}+\varepsilon, & b_{1}^{\left(j_{2}\right)}(\varepsilon)=1, \quad b_{i}^{\left(j_{2}\right)}(\varepsilon)=0,2 \leq i<\lambda_{j_{2}} .
\end{array}
$$

Hence $\left(\iota_{\lambda}^{-1} \circ R_{g_{\lambda \rightarrow \mu}(\varepsilon)} \circ \iota_{\mu}\right)(c)$ is of Jordan's normal form with $l$ distinct eigenvalues $b^{(k)}(k \neq$ $\left.j_{1}, j_{2}\right), b^{(j)}, b^{(j)}+\varepsilon$ of multiplicities $\lambda_{k}\left(k \neq j_{1}, j_{2}\right), \lambda_{j_{1}}, \lambda_{j_{2}}$ for $0<|\varepsilon|<<1$, which implies $\sigma_{\varepsilon}(b) \in B_{\lambda}$. The property $\sigma_{\varepsilon}(b) \in\left(\operatorname{Ad} g_{b} g_{\lambda \rightarrow \mu}(\varepsilon)\right) \mathfrak{h}_{\lambda}$ follows from its definition. The equation (5.1) is derived from $b=\left(\operatorname{Ad} g_{b}\right) c$ and

$$
\lim _{\varepsilon \rightarrow 0}\left(\left(\operatorname{Ad} g_{\lambda \rightarrow \mu}(\varepsilon)\right) \circ \iota_{\lambda}^{-1} \circ R_{g_{\lambda \rightarrow \mu}(\varepsilon)} \circ \iota_{\mu}\right)(c)=c,
$$

which is verified by Lemma 4.2 .

Theorem 5.2 (Convergence of Lie algebras). Let $b \in B_{\mu}$ and $\sigma_{\varepsilon}(b)$ be those given in Theorem 5.1, and let $\lambda \rightarrow \mu$. For any $X \in \mathfrak{h}_{b}$, define $\Psi_{\varepsilon}(X)$ by

$$
\Psi_{\varepsilon}(X):=\left(\left(\operatorname{Ad} g_{b} g_{\lambda \rightarrow \mu}(\varepsilon)\right) \circ \iota_{\lambda}^{-1} \circ R_{g_{\lambda \rightarrow \mu}(\varepsilon)} \circ \iota_{\mu} \circ\left(\operatorname{Ad} g_{b}\right)^{-1}\right)(X) .
$$

Then $\Psi_{\varepsilon}$ is a Lie algebra isomorphism from $\mathfrak{h}_{b}$ to $\mathfrak{h}_{\sigma_{\varepsilon}(b)}$ satisfying

$$
\lim _{\varepsilon \rightarrow 0} \Psi_{\varepsilon}(X)=X, \quad X \in \mathfrak{h}_{b} .
$$

Proof. As in the proof of Theorem 5.1, we can verify that $\Psi_{\varepsilon}(X) \in\left(\operatorname{Ad} g_{b} g_{\lambda \rightarrow \mu}(\varepsilon)\right) \mathfrak{h}_{\lambda}$, which imples $\Psi_{\varepsilon}(X) \in \mathfrak{h}_{\sigma_{\varepsilon}(b)}$ and (5.2). Since $\Psi_{\varepsilon}$ is a linear isomorphism and both $\mathfrak{h}_{b}$ and $\mathfrak{h}_{\sigma_{\varepsilon}(b)}$ are abelian, it is a Lie algebra isomorphism.

It would be better to explain how we understand the construction of $\sigma_{\varepsilon}(b)$ in Theorem 5.1 in the picture of fibration structure $F_{\lambda}: B_{\lambda} \rightarrow Y_{\lambda}$ for the stratum $B_{\lambda}$ of the stratification of $B$ explained in Section 3.

Let us restrict ourselves to the case where $\lambda=(p, q), \mu=(n)=(p+q)$ and $b \in \mathfrak{h}_{\mu}$ is of Jordan's normal form

$$
b=b^{(1)} I_{n}+\Lambda_{n}
$$

First we formed

$$
b_{\varepsilon}:=\left(\iota_{\lambda}^{-1} \circ R_{g_{\lambda \rightarrow \mu}(\varepsilon)} \circ \iota_{\mu}\right)(b)=\left(b^{(1)} I_{p}+\Lambda_{p}\right) \oplus\left(\left(b^{(1)}+\varepsilon\right) I_{q}+\Lambda_{q}\right) .
$$

For any $\varepsilon \neq 0, b_{\varepsilon}$ is a regular element belonging to $B_{\lambda}$. Let $y_{0}=F(b) \in Y_{\mu}$ and $y_{\varepsilon}=$ $F\left(b_{\varepsilon}\right) \in Y_{\lambda} \quad(\varepsilon \neq 0)$. Then $y_{\varepsilon}$ defines a holomorphic curve $\varepsilon \mapsto y_{\varepsilon}$ in $Y_{\lambda}$ which tends to $y_{0} \in Y_{\mu}$ as $\varepsilon \rightarrow 0$. But $b_{\varepsilon}$, which is a lift of the curve $y_{\varepsilon}$ and defines a holomorphic curve in $B_{\lambda}$, tends not to the regular element $b$ but to a subregular element $\left(b^{(1)} I_{p}+\Lambda_{p}\right) \oplus\left(b^{(1)} I_{q}+\Lambda_{q}\right)$ 


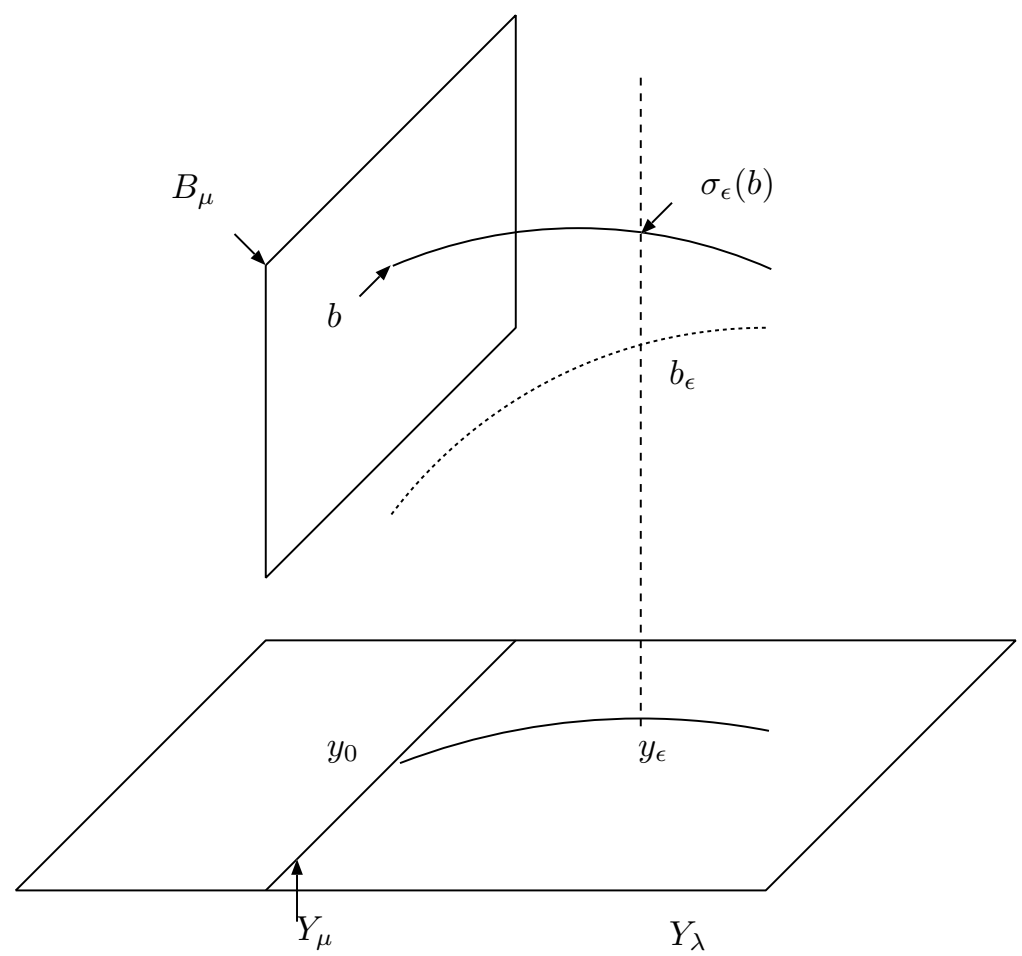

Figure 1. Curve $\sigma_{\varepsilon}$

as $\varepsilon \rightarrow 0$. So we move $b_{\varepsilon}$ in the fiber $F^{-1}\left(y_{\varepsilon}\right)$ by twisting it by the action $\operatorname{Ad} g_{\lambda \rightarrow \mu}(\varepsilon)$. This process defines a desired holomorphic curve $\varepsilon \mapsto \sigma_{\varepsilon}(b)$ in $B_{\lambda}$, which is a lift of holomorphic curve $\varepsilon \mapsto y_{\varepsilon}$ in $Y_{\lambda}$ and satisfies (5.1) (see Fig.1).

Theorem 5.2 says that the same limit process works well not only for a regular element $b \in B_{\mu}$ but also for any elements of Lie algebra $\mathfrak{h}_{b}$ obtained as a centralizer of regular element $b \in B_{\mu}$.

5.2. Conflucence of GHG systems. Let $\Psi_{\varepsilon}^{*}: \mathfrak{h}_{\sigma_{\varepsilon}(b)}^{*} \rightarrow \mathfrak{h}_{b}^{*}$ be the dual isomorphism of $\Psi_{\varepsilon}: \mathfrak{h}_{b} \rightarrow \mathfrak{h}_{\sigma_{\varepsilon}(b)}$. Then we have the following theorem, which is the first main assertion of this paper.

Theorem 5.3 (Confluence of GHG systems). Suppose $\mu \in \mathbf{Y}_{n}$ is adjacent to $\lambda \in \mathbf{Y}_{n}$. Given a GHG system $\mathcal{I}\left(\alpha ; \mathfrak{h}_{b}\right)$ for $b \in B_{\mu}$ and $\alpha \in \mathfrak{h}_{b}^{*}$ with $\left\langle\alpha, I_{n}\right\rangle=-r$, consider the $G H G$ system $\mathcal{I}\left(\alpha(\varepsilon) ; \mathfrak{h}_{\lambda}\right)$, where

$$
\alpha(\varepsilon)=\left(\left(\operatorname{Ad} g_{b} g_{\lambda \rightarrow \mu}(\varepsilon)\right)^{*} \circ\left(\Psi_{\varepsilon}^{*}\right)^{-1}\right)(\alpha) \in \mathfrak{h}_{\lambda}^{*} .
$$

Then the change of variables

$$
z=w g_{b} g_{\lambda \rightarrow \mu}(\varepsilon)
$$

transforms the system $\mathcal{I}\left(\alpha(\varepsilon) ; \mathfrak{h}_{\lambda}\right)$ in $z$ to $\mathcal{I}\left(\left(\Psi_{\varepsilon}^{*}\right)^{-1}(\alpha) ; \mathfrak{h}_{\sigma_{\varepsilon}(b)}\right)$ in w, namely,

$$
\left(R_{\left.\left(g_{b} g_{\lambda \rightarrow \mu}(\varepsilon)\right)^{-1}\right)_{*}} \mathcal{I}\left(\alpha(\varepsilon) ; \mathfrak{h}_{\lambda}\right)=\mathcal{I}\left(\left(\Psi_{\varepsilon}^{*}\right)^{-1}(\alpha) ; \mathfrak{h}_{\sigma_{\varepsilon}(b)}\right)\right.
$$


and

$$
\lim _{\varepsilon \rightarrow 0} \mathcal{I}\left(\left(\Psi_{\varepsilon}^{*}\right)^{-1}(\alpha) ; \mathfrak{h}_{\sigma_{b}(\varepsilon)}\right)=\mathcal{I}\left(\alpha ; \mathfrak{h}_{b}\right)
$$

in the sense that a set of generators of the ideal $\mathcal{I}\left(\alpha ; \mathfrak{h}_{b}\right)$ are obtained as limits of a set of generators of $\mathcal{I}\left(\left(\Psi_{\varepsilon}^{*}\right)^{-1}(\alpha) ; \mathfrak{h}_{\sigma_{b}(\varepsilon)}\right)$ as $\varepsilon \rightarrow 0$.

Remark 5.1. In the case where $r=2, n=4, \lambda=(1,1,1,1)$ and $\mu=(2,1,1)$, the theorem with $g_{b}=I_{4}$ reduces to the confluence process from Gauss to Kummmer explained in the introduction. Indeed, the system $\mathcal{I}\left(\beta ; \mathfrak{h}_{\lambda}\right)$ with $\beta \in \mathfrak{h}_{\lambda}^{*},\left\langle\beta, I_{n}\right\rangle=-2$, the change of variables and parameters $z=w g_{\lambda \rightarrow \mu}(\varepsilon), \beta=\alpha(\varepsilon)$, the system $\mathcal{I}\left(\left(\Psi_{\varepsilon}^{*}\right)^{-1}(\alpha) ; \mathfrak{h}_{\sigma_{\varepsilon}(b)}\right)$, and the system $\mathcal{I}\left(\alpha ; \mathfrak{h}_{b}\right)$ reduce to (1.2), (1.3), (1.4), and (1.5), respectively (see also Section $8)$.

In order to prove the first part of Theorem 5.3, we first show the following lemma.

Lemma 5.1. Let $b \in B_{\lambda}$ and $\alpha \in \mathfrak{h}_{b}^{*}$ with $\left\langle\alpha, I_{n}\right\rangle=-r$. Then, for any $g \in \operatorname{GL}(n, \mathbb{C})$, the change of variables $z=w g$ transforms the system $\mathcal{I}\left(\alpha ; \mathfrak{h}_{b}\right)$ in $z$ to the system $\mathcal{I}\left(\left(\operatorname{Ad} g^{-1}\right)^{*}(\alpha) ; \mathfrak{h}_{(\operatorname{Ad} g) b}\right)$ in w, namely,

$$
\left(R_{g^{-1}}\right)_{*} \mathcal{I}\left(\alpha ; \mathfrak{h}_{b}\right)=\mathcal{I}\left(\left(\operatorname{Ad} g^{-1}\right)^{*}(\alpha) ; \mathfrak{h}_{(\operatorname{Ad} g) b}\right) .
$$

Proof of Lemma 5.1. Recalling that $\partial_{z}$ and $\partial_{w}$ are the matrices whose $(i, j)$ entry is $\partial / \partial z_{i j}$ and $\partial / \partial w_{i j}$, respectively, we have

$$
{ }^{t} \partial_{z}=g^{-1 t} \partial_{w}
$$

Therefore the mapping $R_{g^{-1}}$ takes the generators $L_{X}\left(X \in \mathfrak{h}_{b}\right), M_{Y}(Y \in \mathfrak{g l}(r, \mathbb{C}))$ and $\square_{i i^{\prime}, j j^{\prime}}$ of the ideal $\mathcal{I}\left(\mathfrak{h}_{b} ; \alpha\right)$ to

$$
\begin{aligned}
\left(R_{g^{-1}}\right)_{*} L_{X} & =\operatorname{Tr}\left(w g X g^{-1 t} \partial_{w}\right)-\langle\alpha, X\rangle \\
& =\operatorname{Tr}\left(w((\operatorname{Ad} g) X)^{t} \partial_{w}\right)-\left\langle\left(\operatorname{Ad} g^{-1}\right)^{*} \alpha,(\operatorname{Ad} g) X\right\rangle \\
\left(R_{g^{-1}}\right)_{*} M_{Y} & =\operatorname{Tr}\left(Y w g g^{-1} \partial_{w}+Y\right)=\operatorname{Tr}\left(Y w^{t} \partial_{w}+Y\right)
\end{aligned}
$$

and

$$
\left(R_{g^{-1}}\right)_{*} \square_{i i^{\prime}, j j^{\prime}}=\sum_{k, k^{\prime}}\left(\left(g^{-1}\right)_{j k}\left(g^{-1}\right)_{j^{\prime} k^{\prime}}-\left(g^{-1}\right)_{j k^{\prime}}\left(g^{-1}\right)_{j^{\prime} k}\right) \partial_{w, i k} \partial_{w, i^{\prime} k^{\prime}}
$$

Hence it is sufficient to show that the elements $\left(R_{g^{-1}}\right)_{*} \square_{i i^{\prime}, j j^{\prime}}\left(0 \leq i, i^{\prime}<r, 0 \leq j, j^{\prime}<n\right)$ generate the same ideal of the Weyl algebra on $Z$ as

$$
\square_{w, i i^{\prime}, j j^{\prime}}=\partial_{w, i j} \partial_{w, i^{\prime} j^{\prime}}-\partial_{w, i j^{\prime}} \partial_{w, i^{\prime} j}, \quad 0 \leq i, i^{\prime}<r, 0 \leq j, j^{\prime}<n .
$$

Take arbitrary indices $a, a^{\prime}\left(a \neq a^{\prime}\right)$. Multiplying both sides on (5.3) by $g_{a j} g_{a^{\prime} j^{\prime}}$ and summing them up with respect to $j, j^{\prime}$, we have

$$
\begin{aligned}
\sum_{j, j^{\prime}} g_{a j} g_{a^{\prime} j^{\prime}}\left(R_{g^{-1}}\right)_{*} \square_{i i^{\prime}, j j^{\prime}} & =\sum_{k, k^{\prime}}\left(\delta_{a k} \delta_{a^{\prime} k^{\prime}}-\delta_{a k^{\prime}} \delta_{a^{\prime} k}\right) \partial_{w, i k} \partial_{w, i^{\prime} k^{\prime}} \\
& =\partial_{w, i a} \partial_{w, i^{\prime} a^{\prime}}-\partial_{w, i a^{\prime}} \partial_{w, i^{\prime} a}=\square_{w, i i^{\prime}, a a^{\prime}}
\end{aligned}
$$


Proof of Theorem 5.3. Since the first half of the theorem is obtained by Lemma 5.1, we have only to show the second half.

As generators of the ideal $\mathcal{I}\left(\alpha ; \mathfrak{h}_{b}\right)$, we take $L_{X}\left(X \in \mathfrak{h}_{b}\right), M_{Y}(Y \in \mathfrak{g l}(r, \mathbb{C}))$ and $\square_{i i^{\prime}, j j^{\prime}}\left(0 \leq i, i^{\prime}<r, 0 \leq j, j^{\prime}<n\right)$. Since the mapping $\Psi_{\varepsilon}: \mathfrak{h}_{b} \rightarrow \mathfrak{h}_{\sigma_{\varepsilon}(b)}$ is a Lie algebra isomorphism, we can choose, as generators of the ideal $\mathcal{I}\left(\left(\Psi_{\varepsilon}^{*}\right)^{-1}(\alpha) ; \mathfrak{h}_{\sigma_{\varepsilon}(b)}\right)$, the elements

$$
L_{X}(\varepsilon):=\operatorname{Tr}\left(z \Psi_{\varepsilon}(X)^{t} \partial_{z}\right)-\left\langle\Psi_{\varepsilon}(X),\left(\Psi_{\varepsilon}^{*}\right)^{-1}(\alpha)\right\rangle, \quad X \in \mathfrak{h}_{b},
$$

$M_{Y}, Y \in \mathfrak{g l}(r, \mathbb{C})$ and $\square_{i i^{\prime}, j j^{\prime}}, 0 \leq i, i^{\prime}<r, 0 \leq j, j^{\prime}<n$. Therefore it is sufficient to show that

$$
\lim _{\varepsilon \rightarrow 0} L_{X}(\varepsilon)=L_{X}, \quad X \in \mathfrak{h}_{b},
$$

and this follows from $\lim _{\varepsilon \rightarrow 0} \Psi_{\varepsilon}(X)=X$ (Theorem 5.2) and the trivial equation

$$
\left\langle\left(\Psi_{\varepsilon}^{*}\right)^{-1}(\alpha), \Psi_{\varepsilon}(X)\right\rangle=\langle\alpha, X\rangle .
$$

\section{Confluence of integral Representations}

6.1. Convergence of integrands. In this section, we show that the process of confluence of GHG systems given in Section 5 can be lifted to the convergence of integrands of integral representations.

Let $\lambda, \mu \in \mathbf{Y}_{n}$ such that $\mu$ is adjacent to $\lambda$. For $b \in B_{\mu}$, we define an isomorphism $\psi_{\varepsilon}: \tilde{H}_{b} \rightarrow \tilde{H}_{\sigma_{\varepsilon}(b)}$ so that the following diagram commutes

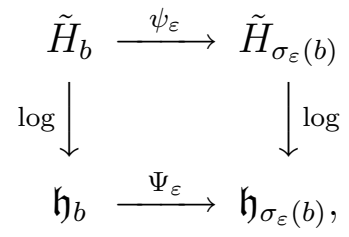

namely,

$$
\psi_{\varepsilon}(h)=(\log )^{-1}\left(\Psi_{\varepsilon}(\log h)\right), \quad h \in \tilde{H}_{\sigma_{\varepsilon}(b)} .
$$

We first note

Theorem 6.1 (Convergence of Lie groups). Suppose $\lambda \rightarrow \mu$. Then, for any $h \in \tilde{H}_{b}$, we have

$$
\lim _{\varepsilon \rightarrow 0} \psi_{\varepsilon}(h)=h
$$

Proof. Take $X \in \mathfrak{h}_{b}$ such that $X=\log h$. Then, by Theorem 5.2, we have

$$
\lim _{\varepsilon \rightarrow 0} \psi_{\varepsilon}(h)=\lim _{\varepsilon \rightarrow 0}(\log )^{-1}\left(\left(\Psi_{\varepsilon}(X)\right)=\log ^{-1}(X)=h .\right.
$$

Concerning a character $\chi_{\sigma_{\varepsilon}(b)}$ of $\tilde{H}_{\sigma_{\varepsilon}(b)}$, we notice the following trivial property.

Theorem 6.2. For any $h \in \tilde{H}_{b}$ and $\alpha \in \mathfrak{h}_{b}^{*}$, it holds that

$$
\chi_{\sigma_{\varepsilon}(b)}\left(\left(\Psi_{\varepsilon}^{*}\right)^{-1}(\alpha) ; \psi_{\varepsilon}(h)\right)=\chi_{b}(\alpha ; h), \quad 0<|\varepsilon|<<1 .
$$


Proof. Let $X=\log h \in \mathfrak{h}_{b}$. Then we have

$$
\chi_{b}(\alpha ; h)=\exp (\langle\alpha, X\rangle), \quad \chi_{\sigma_{\varepsilon}(b)}\left(\left(\Psi_{\varepsilon}^{*}\right)^{-1}(\alpha) ; \psi_{\varepsilon}(h)\right)=\exp \left(\left\langle\left(\Psi_{\varepsilon}^{*}\right)^{-1}(\alpha), \Psi_{\varepsilon}(X)\right\rangle\right) .
$$

Hence the theorem is derived from $\left\langle\left(\Psi_{\varepsilon}^{*}\right)^{-1}(\alpha), \Psi_{\varepsilon}(X)\right\rangle=\langle\alpha, X\rangle$.

Now we give the following theorem, which is the latter part of our main assertion of this paper.

Theorem 6.3 (Convergence of integrands). Suppose that $\lambda \rightarrow \mu, b \in B_{\mu}$ and $\alpha \in \mathfrak{h}_{b}^{*}$. Then we have

$$
\lim _{\varepsilon \rightarrow 0} \chi_{\sigma_{\varepsilon}(b)}\left(\left(\Psi_{\varepsilon}^{*}\right)^{-1}(\alpha) ; \iota_{\sigma_{\varepsilon}(b)}^{-1}\left(\iota_{b}(h)\right)\right)=\chi_{b}(\alpha ; h)
$$

for any $h \in \tilde{H}_{b}$, and hence we have

$$
\lim _{\varepsilon \rightarrow 0} \chi_{\sigma_{\varepsilon}(b)}\left(\left(\Psi_{\varepsilon}^{*}\right)^{-1}(\alpha) ; \iota_{\sigma_{\varepsilon}(b)}^{-1}(t z)\right)=\chi_{b}\left(\alpha ; \iota_{b}^{-1}(t z)\right) .
$$

Remark 6.1. We remark that in Theorem 6.3

$$
\chi_{\sigma_{\varepsilon}(b)}\left(\left(\Psi_{\varepsilon}^{*}\right)^{-1}(\alpha) ; \iota_{\sigma_{\varepsilon}(b)}^{-1}\left(\iota_{b}(h)\right)\right) \neq \chi_{b}(\alpha ; h)
$$

for general $h \in \tilde{H}_{b}$. This fact does not contradict Theorem 6.2 , because $\iota_{\sigma_{\varepsilon}(b)}^{-1}\left(\iota_{b}(h)\right) \neq$ $\psi_{\varepsilon}(h)$, although

$$
\iota_{\sigma_{\varepsilon}(b)}^{-1}\left(\iota_{b}(h)\right)=\psi_{\varepsilon}(h)\left(I_{n}+O(\varepsilon)\right) .
$$

We omit the proof of (6.2), since it can be verified by the same argument as in the next subsection.

6.2. Proof of Theorem 6.3. We first show a lemma which will be used in proving Theorem 6.3.

Lemma 6.1. Let $x=\left(x_{0}, x_{1}, \ldots\right)$ and let $y(x, t)=\left(y_{0}(x, t), y_{1}(x, t), \ldots\right)$ be a sequence of formal power series of $t$ defined by

$$
y_{i}(x, t)=\sum_{k \geq 0}\left(\begin{array}{c}
i+k \\
k
\end{array}\right) x_{i+k} t^{k}, \quad i \geq 0 .
$$

Then we have

$$
\theta_{i}\left(y_{0}(x, t), y_{1}(x, t), \ldots\right)=\sum_{k \geq 0}\left(\begin{array}{c}
i+k \\
k
\end{array}\right) \theta_{i+k}\left(x_{0}, x_{1}, \ldots\right) t^{k}, \quad i \geq 0,
$$

where $\theta_{i}$ are functions defined by (2.6).

Proof. Denote by $f(x, t)$ the formal power series $\sum_{k \geq 0} x_{k} t^{k}$. Then

$$
y_{i}(x, t)=(1 / i !)(d / d t)^{i} f(x, t) .
$$

Therefore, we have

$$
f(y(x, t), s)=\sum_{i \geq 0}(1 / i !)(d / d t)^{i} f(x, t) s^{i}=e^{s(d / d t)} f(x, t)=f(x, t+s) .
$$

By expanding both sides of $\log f(y(x, t), s)=\log f(x, t+s)$ into formal power series of $s$, and by comparing the coefficients of $s^{i}$, we obtain the desired result. 
Proof of Theorem 6.3. Let $\mu_{j}=\lambda_{j_{1}}+\lambda_{j_{2}}$ for $0 \leq j<\ell(\mu), 0 \leq j_{2}<j_{1}<\ell(\lambda)$. Since $\sigma_{\varepsilon}(b) \in\left(\operatorname{Ad} g_{b} g_{\lambda \rightarrow \mu}(\varepsilon)\right) \mathfrak{h}_{\lambda}$, we have

$$
\begin{aligned}
& \chi_{\sigma_{\varepsilon}(b)}\left(\left(\Psi_{\varepsilon}^{*}\right)^{-1}(\alpha) ; \iota_{\sigma_{\varepsilon}(b)}^{-1}\left(\iota_{b}(h)\right)\right) \\
& \quad=\chi_{\lambda}\left(\left(\operatorname{Ad} g_{b} g_{\lambda \rightarrow \mu}(\varepsilon)\right)^{*}\left(\Psi_{\varepsilon}^{*}\right)^{-1}(\alpha) ;\left(\operatorname{Ad} g_{b} g_{\lambda \rightarrow \mu}(\varepsilon)\right)^{-1} \iota_{\sigma_{\varepsilon}(b)}^{-1}\left(\iota_{b}(h)\right)\right) .
\end{aligned}
$$

We can verify that

$$
\begin{aligned}
\left(\left(\operatorname{Ad} g_{b} g_{\lambda \rightarrow \mu}(\varepsilon)\right)^{*} \circ\left(\Psi_{\varepsilon}^{*}\right)^{-1}\right)(\alpha) & =\left(f_{\varepsilon}^{-1}\right)^{*}\left(\operatorname{Ad} g_{b}\right)^{*}(\alpha), \\
\left(\operatorname{Ad} g_{b} g_{\lambda \rightarrow \mu}(\varepsilon)\right)^{-1} \iota_{\sigma_{\varepsilon}(b)}^{-1}\left(\iota_{b}(h)\right) & =\left(\left(f_{\varepsilon}\right) \circ\left(\operatorname{Ad} g_{b}\right)^{-1}\right)(h),
\end{aligned}
$$

where

$$
f_{\varepsilon}:=\iota_{\lambda}^{-1} \circ R_{g_{\lambda \rightarrow \mu}(\varepsilon)} \circ \iota_{\mu} .
$$

Notice that by the same letter $f_{\varepsilon}$, we express an isomorphism $f_{\varepsilon}: \mathfrak{h}_{\mu} \rightarrow \mathfrak{h}_{\lambda}$ and a mapping $f_{\varepsilon}: \tilde{H}_{\mu} \rightarrow \tilde{H}_{\lambda}$. We have also

$$
\chi_{b}(\alpha ; h)=\chi_{\mu}\left(\left(\operatorname{Ad} g_{b}\right)^{*}(\alpha) ;\left(\operatorname{Ad} g_{b}\right)^{-1} h\right),
$$

since $b \in\left(\operatorname{Ad} g_{b}\right) \mathfrak{h}_{\mu}$. Therefore (6.1) is equivalent to

$$
\lim _{\varepsilon \rightarrow 0} \chi_{\lambda}\left(\left(f_{\varepsilon}^{-1}\right)^{*}(\alpha) ; f_{\varepsilon}(h)\right)=\chi_{\lambda}(\alpha ; h)
$$

for any $\alpha \in \mathfrak{h}_{\mu}^{*}$ and $h \in \tilde{H}_{\mu}$. Set $\lambda^{\prime}:=\left(\lambda_{j_{1}}, \lambda_{j_{2}}\right) \in \mathbf{Y}_{\mu_{j}}, \mu^{\prime}=\left(\mu_{j}\right) \in \mathbf{Y}_{\mu_{j}}, \alpha^{\prime}:=\left.\alpha\right|_{\mathfrak{j}\left(\mu_{j}\right)}$ and denote by $h^{\prime} \in \tilde{J}\left(\mu_{j}\right)$ the $j$-th component of $h \in \tilde{H}_{\mu}$. We also set $f_{\varepsilon}^{\prime}:=\iota_{\lambda^{\prime}}^{-1} \circ R_{g^{(j)}(\varepsilon)} \circ \iota_{\mu^{\prime}}$. Then we can verify

$$
\chi_{\lambda}\left(\left(f_{\varepsilon}^{-1}\right)^{*}(\alpha) ; f_{\varepsilon}(h)\right) / \chi_{\lambda}(\alpha ; h)=\chi_{\lambda^{\prime}}\left(\left(f_{\varepsilon}^{\prime-1}\right)^{*}\left(\alpha^{\prime}\right) ; f_{\varepsilon}^{\prime}\left(h^{\prime}\right)\right) / \chi_{\mu^{\prime}}\left(\alpha^{\prime} ; h^{\prime}\right) .
$$

Therefore we have only to prove (6.3) in the case where $\lambda=(p, q)$ and $\mu=(p+q)$ with $p+q=n$.

Hereafter we suppose $\lambda=(p, q), \mu=(p+q)=(n)$ and $f_{\varepsilon}=\iota_{\lambda}^{-1} \circ R_{g_{\lambda \rightarrow \mu}(\varepsilon)} \circ \iota_{\mu}$. For $h=\sum_{0 \leq i<p+q} h_{i} \Lambda_{p+q}^{i}$, put

$$
f_{\varepsilon}(h)=\left(\sum_{0 \leq i<p} h_{i}^{\prime}(\varepsilon) \Lambda_{p}^{i}\right) \oplus\left(\sum_{0 \leq i<q} h_{i}^{\prime \prime}(\varepsilon) \Lambda_{q}^{i}\right) .
$$

We notice that $h_{i}^{\prime}(\varepsilon)=h_{i}, 0 \leq i<p$ and

$$
h_{i}^{\prime \prime}(\varepsilon)=\sum_{k \geq 0}\left(\begin{array}{c}
i+k \\
i
\end{array}\right) h_{i+k} \varepsilon^{k}, \quad 0 \leq i<q .
$$

For $\alpha \in \mathfrak{h}_{\mu}^{*}$, set

$$
\begin{aligned}
\alpha_{i} & :=\left\langle\alpha, \Lambda_{p+q}^{i}\right\rangle, 0 \leq i<p+q, \\
\alpha_{i}^{\prime}(\varepsilon) & :=\left\langle\left(f_{\varepsilon}^{-1}\right)^{*}(\alpha), \Lambda_{p}^{i} \oplus O_{q}\right\rangle, 0 \leq i<p, \\
\alpha_{i}^{\prime \prime}(\varepsilon) & :=\left\langle\left(f_{\varepsilon}^{-1}\right)^{*}(\alpha), O_{p} \oplus \Lambda_{q}^{i}\right\rangle, 0 \leq i<q,
\end{aligned}
$$

where $O_{q}$ and $O_{p}$ are zero matrices of size $q$ and $p$, respectively. We see that

$$
\left(\alpha_{0}^{\prime}(\varepsilon), \ldots, \alpha_{p}^{\prime}(\varepsilon), \alpha_{0}^{\prime \prime}(\varepsilon), \ldots, \alpha_{q}^{\prime \prime}(\varepsilon)\right)^{t} g_{\lambda \rightarrow \mu}(\varepsilon)=\left(\alpha_{0}, \ldots, \alpha_{p+q}\right) .
$$


Therefore we have

(6.6) $\log \chi_{\lambda}\left(\left(f_{\varepsilon}^{-1}\right)^{*}(\alpha) ; f_{\varepsilon}(h)\right)=\sum_{0 \leq i<p} \alpha_{i}^{\prime}(\varepsilon) \theta_{i}\left(h_{0}, h_{1}, \ldots\right)+\sum_{0 \leq i<q} \alpha_{i}^{\prime \prime}(\varepsilon) \theta_{i}\left(h_{0}^{\prime \prime}(\varepsilon), h_{1}^{\prime \prime}(\varepsilon), \ldots\right)$.

From (6.4) and Lemma 6.1, it follows that

$$
\theta_{i}\left(h_{0}^{\prime \prime}(\varepsilon), h_{1}^{\prime \prime}(\varepsilon), \ldots\right)=\sum_{k \geq 0}\left(\begin{array}{c}
i+k \\
k
\end{array}\right) \theta_{i+k}\left(h_{0}, h_{1}, \ldots\right) \varepsilon^{k}, \quad 0 \leq i<q .
$$

Substituting (6.7) into (6.6), we obtain

$$
\begin{aligned}
\log \chi_{\lambda}= & \sum_{0 \leq i<p}\left(\alpha_{i}^{\prime}(\varepsilon)+\sum_{0 \leq k<q}\left(\begin{array}{l}
i \\
k
\end{array}\right) \alpha_{k}^{\prime \prime}(\varepsilon) \varepsilon^{i-k}\right) \theta_{i}\left(h_{0}, h_{1}, \ldots\right) \\
& +\sum_{p \leq i<p+q}\left(\sum_{0 \leq k<q}\left(\begin{array}{l}
i \\
k
\end{array}\right) \alpha_{k}^{\prime \prime}(\varepsilon) \varepsilon^{i-k}\right) \theta_{i}\left(h_{0}, h_{1}, \ldots\right)+O(\varepsilon) .
\end{aligned}
$$

Here we notice that the relation (6.5) means

$$
\begin{aligned}
\alpha_{i}^{\prime}(\varepsilon)+ & \sum_{0 \leq k<q}\left(\begin{array}{l}
i \\
k
\end{array}\right) \alpha_{k}^{\prime \prime}(\varepsilon) \varepsilon^{i-k}=\alpha_{i}, \quad 0 \leq i<p, \\
& \sum_{0 \leq k<q}\left(\begin{array}{l}
i \\
k
\end{array}\right) \alpha_{k}^{\prime \prime}(\varepsilon) \varepsilon^{i-k}=\alpha_{i}, \quad p \leq i<p+q .
\end{aligned}
$$

Therefore we get

$$
\log \chi_{\lambda}\left(\left(f_{\varepsilon}^{-1}\right)^{*}(\alpha) ; f_{\varepsilon}(h)\right)=\sum_{0 \leq i<p+q} \alpha_{i} \theta_{i}\left(h_{0}, h_{1}, \ldots\right)+O(\varepsilon)=\log \chi_{\mu}(\alpha ; h)+O(\varepsilon) .
$$

Thus we have completed the proof of Theorem 6.3.

\section{Proof of Lemma 4.1}

Denote by $A_{12}$ the $p \times q$ matrix whose $(i, j)$ entry $(0 \leq i<p, 0 \leq j<q)$ is $\left(\begin{array}{l}i \\ j\end{array}\right)$ and by $A_{22}$ the $q \times q$ matrix whose $(i, j)$ entry $(0 \leq i, j<q)$ is $\left(\begin{array}{c}p+i \\ j\end{array}\right)$. Then we have

$$
G_{12}=D_{p}(\varepsilon) A_{12} D_{q}\left(\varepsilon^{-1}\right), \quad G_{22}=\varepsilon^{p} D_{q}(\varepsilon) A_{22} D_{q}\left(\varepsilon^{-1}\right) .
$$

Set

$$
Y_{11}=\sum_{0 \leq i<p} y_{i}(\varepsilon) \Lambda_{p}^{i}\left(=\sum_{0 \leq i<p} x_{i} \Lambda_{p}^{i}\right), \quad Y_{22}=\sum_{p \leq i<p+q} y_{i}(\varepsilon) \Lambda_{q}^{i-p} .
$$

Then

$$
(\operatorname{Ad} g(\varepsilon)) Y(\varepsilon)=\left(\begin{array}{cc}
Y_{11} & \left(-Y_{11} G_{12}+G_{12} Y_{22}\right) G_{22}^{-1} \\
0 & G_{22} Y_{22} G_{22}^{-1}
\end{array}\right) .
$$

We first obtain $\lim _{\varepsilon \rightarrow 0} G_{22} Y_{22} G_{22}^{-1}$. From (4.1), (4.2) and (4.3), we have

$$
Y_{22}=\sum_{p \leq i<p+q} y_{i}(\varepsilon) \Lambda_{q}^{i-p}=\sum_{p \leq i<p+q} \sum_{0 \leq k<p+q}\left(\begin{array}{c}
k \\
i-p
\end{array}\right) x_{k} \varepsilon^{k-i+p} \Lambda_{q}^{i-p} .
$$


Then, from $\varepsilon^{-i+p} \Lambda_{q}^{i-p}=D_{q}(\varepsilon) \Lambda_{q}^{i-p} D_{q}\left(\varepsilon^{-1}\right)$, it follows that

$$
Y_{22}=D_{q}(\varepsilon)\left[\sum_{0 \leq k<p+q} x_{k} \varepsilon^{k}\left(I_{q}+\Lambda_{q}\right)^{k}\right] D_{q}\left(\varepsilon^{-1}\right) \text {. }
$$

By (7.1) and (7.3), we have

$$
G_{22} Y_{22} G_{22}^{-1}=\sum_{0 \leq k<m} x_{k} \varepsilon^{k} D_{q}(\varepsilon) A_{22}\left(I_{q}+\Lambda_{q}\right)^{k} A_{22}^{-1} D_{q}\left(\varepsilon^{-1}\right) .
$$

Denote by $A$ the $q \times q$ matrix whose $(i, j)$ entry $(0 \leq i, j<q)$ is $\left(\begin{array}{l}i \\ j\end{array}\right)$. Then we notice that $A$ is a lower triangular matrix with the diagonal entries 1 and its inverse $A^{-1}$ is the matrix whose $(i, j)$ entry is $(-1)^{i+j}\left(\begin{array}{l}i \\ j\end{array}\right)$ (cf. [5], pp465-466). Notice also that, for any integer $m \geq 0$, we have the identity

$$
A\left(I_{q}+\Lambda_{q}\right)^{m}=\left(\left(\begin{array}{c}
i+m \\
j
\end{array}\right)\right)_{0 \leq i, j<q},
$$

which can be seen by virtue of $\left(\begin{array}{l}r \\ l\end{array}\right)+\left(\begin{array}{c}r \\ l-1\end{array}\right)=\left(\begin{array}{c}r+1 \\ l\end{array}\right)$. In particular, we have

$$
A_{22}=A\left(I_{q}+\Lambda_{q}\right)^{p} \text {. }
$$

By (7.6), we have $A_{22}\left(I_{q}+\Lambda_{q}\right)^{k} A_{22}^{-1}=A\left(I_{q}+\Lambda_{q}\right)^{k} A^{-1}$. Noticing that both $A$ and $A^{-1}$ are lower triangular matrices with diagonal entries are 1 , we see that $A_{22}\left(I_{q}+\Lambda_{q}\right)^{k} A_{22}^{-1}$ is the matrix whose entries of the $m$-th upper diagonal $(m>k)$ are all zeros and those of the $k$-th diagonal are 1 . It follows that

$$
G_{22} Y_{22} G_{22}^{-1}=\sum_{0 \leq k<p+q} x_{k}\left[\Lambda_{q}^{k}+O(\varepsilon)\right] \longrightarrow \sum_{0 \leq k<q} x_{k} \Lambda_{q}^{k}
$$

as $\varepsilon \rightarrow 0$.

We next compute $\lim _{\varepsilon \rightarrow 0}\left(-Y_{11} G_{12}+G_{12} Y_{22}\right) G_{22}^{-1}$. By (7.1), (7.2) and (7.3) together with the relation $\Lambda_{p}^{k} D_{p}(\varepsilon)=\varepsilon^{k} D_{p}(\varepsilon) \Lambda_{p}^{k}$, we have

$$
\begin{aligned}
& \left(-Y_{11} G_{12}+G_{12} Y_{22}\right) G_{22}^{-1} \\
& \quad=\sum_{0 \leq k<p+q} x_{k} \varepsilon^{k-p} D_{p}(\varepsilon)\left[-\Lambda_{p}^{k} A_{12}+A_{12}\left(I_{q}+\Lambda_{q}\right)^{k}\right] A_{22}^{-1} D_{q}\left(\varepsilon^{-1}\right) .
\end{aligned}
$$

Let $y_{i j}^{k}$ and $z_{i j}^{k}, \quad(0 \leq i<p, 0 \leq j<q)$ be the $(i, j)$ entry of $\Lambda_{p}^{k} A_{12}$ and $A_{12}\left(I_{q}+\Lambda_{q}\right)^{k}$, respectively. Then

$$
\begin{aligned}
& y_{i j}^{k}=\left\{\begin{array}{cc}
\left(\begin{array}{c}
i+k \\
j
\end{array}\right) & \text { if } i \leq p-k-1, \\
0 & \text { if } i>p-k-1,
\end{array}\right. \\
& z_{i j}^{k}=\left(\begin{array}{c}
i+k \\
j
\end{array}\right),
\end{aligned}
$$

which yields

$$
-y_{i j}^{k}+z_{i j}^{k}= \begin{cases}0 & \text { if } i \leq p-k-1 \\
\left(\begin{array}{c}
i+k \\
j
\end{array}\right) & \text { if } i>p-k-1\end{cases}
$$


Then, by (7.6) and (7.8), we have, for $k \leq p$,

$$
\begin{aligned}
& {\left[-\Lambda_{p}^{k} A_{12}+A_{12}\left(I_{q}+\Lambda_{q}\right)^{k}\right] A_{22}^{-1}=\left(\begin{array}{ccc}
0 & \ldots & 0 \\
\vdots & & \vdots \\
0 & \ldots & 0 \\
\left(\begin{array}{c}
p \\
0
\end{array}\right) & \ldots & \left(\begin{array}{c}
p \\
q-1
\end{array}\right) \\
\vdots & & \vdots \\
\left(\begin{array}{c}
p+k-1 \\
0
\end{array}\right) & \ldots & \left(\begin{array}{c}
p+k-1 \\
q-1
\end{array}\right)
\end{array}\right)\left(I_{q}+\Lambda_{q}\right)^{-p} A^{-1}} \\
& =\left(\begin{array}{ccc}
0 & \cdots & 0 \\
\vdots & & \vdots \\
0 & \cdots & 0 \\
\left(\begin{array}{c}
0 \\
0
\end{array}\right) & \cdots & \left(\begin{array}{c}
0 \\
q-1
\end{array}\right) \\
\vdots & & \vdots \\
\left(\begin{array}{c}
k-1 \\
0
\end{array}\right) & \cdots & \left(\begin{array}{c}
k-1 \\
q-1
\end{array}\right)
\end{array}\right) A^{-1}
\end{aligned}
$$

and, for $k>p$,

$$
\begin{aligned}
& {\left[-\Lambda_{p}^{k} A_{12}+A_{12}\left(I_{q}+\Lambda_{q}\right)^{k}\right] A_{22}^{-1}=A_{12}\left(I_{q}+\Lambda_{q}\right)^{k}\left(I_{q}+\Lambda_{q}\right)^{-p} A^{-1}} \\
& =\left(\begin{array}{ccc}
\left(\begin{array}{c}
0 \\
0
\end{array}\right) & \ldots & \left(\begin{array}{c}
0 \\
q-1
\end{array}\right) \\
\vdots & & \vdots \\
\left(\begin{array}{c}
p-1 \\
0
\end{array}\right) & \ldots & \left(\begin{array}{c}
p-1 \\
q-1
\end{array}\right)
\end{array}\right)\left(I_{q}+\Lambda_{q}\right)^{k-p} A^{-1} .
\end{aligned}
$$

Now, let $w_{i j}^{k}$ be the $(i, j)$ entry of $\left[-\Lambda_{p}^{k} A_{12}+A_{12}\left(I_{q}+\Lambda_{q}\right)^{k}\right] A_{22}^{-1}$. Then, from (7.9) and (7.10) together with the identity $A A^{-1}=I_{q}$, it follows that

$$
w_{i j}^{k}= \begin{cases}1 & \text { if }(j+p)-i=k, \\ 0 & \text { if }(j+p)-i>k .\end{cases}
$$

Denote by $S^{k}$ the $p \times q$ matrix whose $(i, j)$ entry $s_{i j}^{k}(0 \leq i<p, 0 \leq j<q)$ satisfies

$$
s_{i j}^{k}= \begin{cases}1 & \text { if }(j+p)-i=k \\ 0 & \text { otherwise }\end{cases}
$$

Then, by (7.7) and (7.11), we have

$$
\left(-Y_{11} G_{12}+G_{12} Y_{22}\right) G_{22}^{-1}=\sum_{0 \leq k<p+q} x_{k}\left[S^{k}+O(\varepsilon)\right] \longrightarrow \sum_{0 \leq k<p+q} x_{k} S^{k}
$$

as $\varepsilon \rightarrow 0$. Thus we have completed the proof of Lemma 4.1 .

\section{EXAMPLES}

We explain the confluences of the Gauss hypergeometric differential equation described in the diagram (1.1) in Introduction and those of Appell's hypergeometric system $\left(F_{1}\right)$ of two independent variables in the framework of GHG systems. 
First we notice that the GHG functions $u(z)$ for the system $\mathcal{I}\left(\alpha ; \mathfrak{h}_{b}\right)$ satisfy

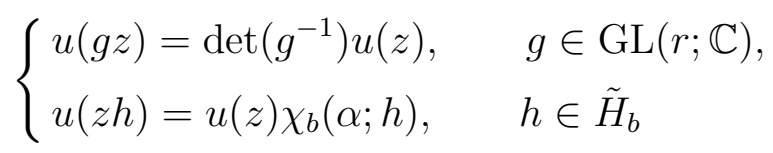

if the branch of $u(z)$ is appropriately chosen.

We recall ([18]) that the Gauss hypergeometric equation and the confluent family in the diagram (1.1) in the Introduction are

(Gauss)

(Kummer)

(Bessel)

(Hermite-Weber)

(Airy)

$$
\begin{aligned}
& x(1-x) u^{\prime \prime}+\{c-(a+b+1) x\} u^{\prime}-a b u=0, \\
& x u^{\prime \prime}+(c-x) u^{\prime}-a u=0, \\
& x^{2} u^{\prime \prime}+x u^{\prime}+\left(x^{2}-\nu^{2}\right) u=0, \\
& u^{\prime \prime}-2 x u^{\prime}+2 \nu u=0, \\
& u^{\prime \prime}-x u=0 .
\end{aligned}
$$

These equations will be denoted by $(\mathrm{G}),(\mathrm{K}),(\mathrm{B}),(\mathrm{H}-\mathrm{W})$ and $(\mathrm{A})$, respectively. The solutions of these differential equations are expressed by the integrals

$$
\begin{aligned}
& u(x)=\int_{C}(1-x t)^{-b}(1-t)^{c-a-1} t^{a-1} d t, \\
& u(x)=\int_{C} e^{x t}(1-t)^{c-a-1} t^{a-1} d t, \\
& u(x)=\int_{C} e^{\frac{x}{2}\left(t-\frac{1}{t}\right)} t^{-\nu-1} d t, \\
& u(x)=\int_{C} e^{2 x t-t^{2}} t^{-\nu-1} d t, \\
& u(x)=\int_{C} e^{x t-\frac{1}{3} t^{3}} d t,
\end{aligned}
$$

when the paths of integration $C$ are appropriately chosen.

Looking at these integral representations, we see that the above equations $(\mathrm{G}),(\mathrm{K})$, (B), (H-W) and (A) correspond to the GHG systems associated with the group $H_{\lambda}$ with the Young diagrams of weight $4: \lambda=(1,1,1,1),(2,1,1),(2,2),(3,1)$ and (4), respectively, 
defined on the matrix spaces $X_{\lambda}$ given by

$$
\begin{aligned}
X_{(1,1,1,1)} & =\left\{\left(\begin{array}{cccc}
1 & 1 & 1 & 0 \\
0 & -x & -1 & 1
\end{array}\right) \mid x \neq 0,1\right\}, \\
X_{(2,1,1)} & =\left\{\left(\begin{array}{cccc}
1 & 0 & 1 & 0 \\
0 & x & -1 & 1
\end{array}\right) \mid x \neq 0\right\}, \\
X_{(2,2)} & =\left\{\left(\begin{array}{cccc}
1 & 0 & 0 & x \\
0 & x & 1 & 0
\end{array}\right) \mid x \neq 0\right\}, \\
X_{(3,1)} & =\left\{\left(\begin{array}{cccc}
1 & 0 & 0 & 0 \\
0 & 1 & \sqrt{2} x & 1
\end{array}\right)\right\}, \\
X_{(4)} & =\left\{\left(\begin{array}{llll}
1 & 0 & 0 & 0 \\
0 & 1 & 0 & x
\end{array}\right)\right\}
\end{aligned}
$$

with the parameters $\alpha_{\lambda} \in \mathfrak{h}_{\lambda}^{*}$ (expressed using the basis of $\mathfrak{h}_{\lambda}$ as in (2.7)):

$$
\begin{aligned}
\alpha_{(1,1,1,1)} & =(b-c,-b, a-1, c-a-1), \\
\alpha_{(2,1,1)} & =(-c, 1, a-1, c-a-1), \\
\alpha_{(2,2)} & =(\nu-1,1,-\nu-1,-1), \\
\alpha_{(3,1)} & =(\nu-1,0,1,-\nu-1), \\
\alpha_{(4)} & =(-2,0,0,1) .
\end{aligned}
$$

Sometimes it is preferable to consider the GHG systems on

$$
\begin{aligned}
& X_{(2,2)}^{\prime}=\left\{\left(\begin{array}{llll}
1 & 0 & 0 & x^{2} / 4 \\
0 & 1 & 1 & 0
\end{array}\right) \mid x \neq 0\right\}, \\
& X_{(3,1)}^{\prime}=\left\{\left(\begin{array}{llll}
1 & 0 & 0 & 0 \\
0 & 1 & x & 1
\end{array}\right)\right\},
\end{aligned}
$$

in place of those on $X_{(2,2)}$ and $X_{(3,1)}$, respectively. Then the differential equations (B) and $(\mathrm{H}-\mathrm{W})$ change their form to

$$
\begin{aligned}
& {\left[\left(\vartheta_{x}+\nu\right)^{2}+x^{2}-\nu^{2}\right] u=0, \quad \vartheta_{x}=x d / d x,} \\
& u^{\prime \prime}-x u^{\prime}+\nu u=0,
\end{aligned}
$$

respectively, and the integral representations of the solutions are

$$
\begin{aligned}
& u(x)=\int_{C} e^{t-\frac{x^{2}}{4 t}} t^{-\nu-1} d t, \\
& u(x)=\int_{C} e^{x t-\frac{1}{2} t^{2}} t^{-\nu-1} d t,
\end{aligned}
$$


respectively. The equation (B') is related to (B) in the following way. In the integral representation for (B), we make a change of integration variable $t \mapsto s=x t / 2$. Then we see that

$$
u(x)=\int_{C} e^{\frac{x}{2}\left(t-\frac{1}{t}\right)} t^{-\nu-1} d t=\left(\frac{x}{2}\right)^{\nu} \int_{C^{\prime}} e^{s-\frac{x^{2}}{4 s}} s^{-\nu-1} d s
$$

and the change of unknown $u \mapsto v$ defined by $u=\left(\frac{x}{2}\right)^{\nu} v$ transforms the equation (B) to (B'). The equation (H-W') is obtained from (H-W) by the simple change of independent variable $x \mapsto x^{\prime}=\sqrt{2} x$.

8.1. Gauss $\rightarrow$ Kummer. In the present and following subsections, we denote by $x$ (resp. $\xi)$ the independent variable of the source equation (resp. target equation) in the process of confluence. Namely, in the present case, $x$ is the variable of $(\mathrm{G})$ and $\xi$ is the variable of $(\mathrm{K})$. We denote by $\tilde{x}$ and $\tilde{\alpha}$ the matrix variable and parameters for the source GHG system corresponding to the source equation, and by $w$ and $\alpha$ those of the target GHG system. In the present case,

$$
\tilde{x}=\left(\begin{array}{cccc}
1 & 1 & 1 & 0 \\
0 & -x & -1 & 1
\end{array}\right) \in X_{(1,1,1,1)}, \quad \tilde{\alpha}=(b-c,-b, a-1, c-a-1)
$$

and

$$
w=\left(\begin{array}{cccc}
1 & 0 & 1 & 0 \\
0 & \xi & -1 & 1
\end{array}\right) \in X_{(2,1,1)}, \quad \alpha=(-c, 1, a-1, c-a-1) .
$$

Then the recipe of the confluence given in Theorem 5.3 is to consider the GHG system of type $(1,1,1,1)$ with the matrix variable $z(\varepsilon)$ and the parametes $\alpha(\varepsilon)$ defined by

$$
\begin{aligned}
& z(\varepsilon)=w g(\varepsilon)=\left(\begin{array}{cccc}
1 & 0 & 1 & 0 \\
0 & \varepsilon \xi & -1 & 1
\end{array}\right), \quad g(\varepsilon)=\left(\begin{array}{cccc}
1 & 1 & & \\
0 & \varepsilon & & \\
& & 1 & \\
& & 1
\end{array}\right), \\
& \alpha(\varepsilon)=\alpha^{t} g(\varepsilon)^{-1}=\left(-c-\varepsilon^{-1}, \varepsilon^{-1}, a-1, c-a-1\right) .
\end{aligned}
$$

Noting that $z(\varepsilon)$ is obtained from $\tilde{x}$ by substituting $-\varepsilon \xi$ in $x$, we have the change of variable and parameters (1.3), which transform the equation $(\mathrm{G})$ to (1.4) and gives the Kummer's equation $(\mathrm{K})$ in the limit $\varepsilon \rightarrow 0$.

8.2. Kummer $\rightarrow$ Bessel. We show the confluence from (K) to (B') instead of $(\mathrm{K}) \rightarrow$ (B). Let

$$
\tilde{x}=\left(\begin{array}{cccc}
1 & 0 & 0 & 1 \\
0 & x & 1 & -1
\end{array}\right) \in X_{(2,1,1)}, \quad \tilde{\alpha}=(-c, 1, a-1, c-a-1)
$$

and

$$
w=\left(\begin{array}{cccc}
1 & 0 & 0 & \left(\frac{\xi}{2}\right)^{2} \\
0 & 1 & 1 & 0
\end{array}\right) \in X_{(2,2)}^{\prime}, \quad \alpha=(\nu-1,1,-\nu-1,-1)
$$


be the data for the GHG systems corresponding to $(\mathrm{K})$ and (B'), respectively. Then the recipe of the confluence is to consider the GHG system of type $(2,1,1)$ with

$$
\begin{aligned}
& z(\varepsilon)=w g(\varepsilon)=\left(\begin{array}{cccc}
1 & 0 & 0 & \left(\frac{\xi}{2}\right)^{2} \varepsilon \\
0 & 1 & 1 & 1
\end{array}\right), \quad g(\varepsilon)=\left(\begin{array}{cccc}
1 & & & \\
0 & 1 & & \\
& & 1 & 1 \\
& & & \varepsilon
\end{array}\right) \\
& \alpha(\varepsilon)=\alpha^{t} g(\varepsilon)^{-1}=\left(\nu-1,1,-\nu-1+\varepsilon^{-1},-\varepsilon^{-1}\right)
\end{aligned}
$$

and relate it to the GHG system for $(\mathrm{K})$. Noting

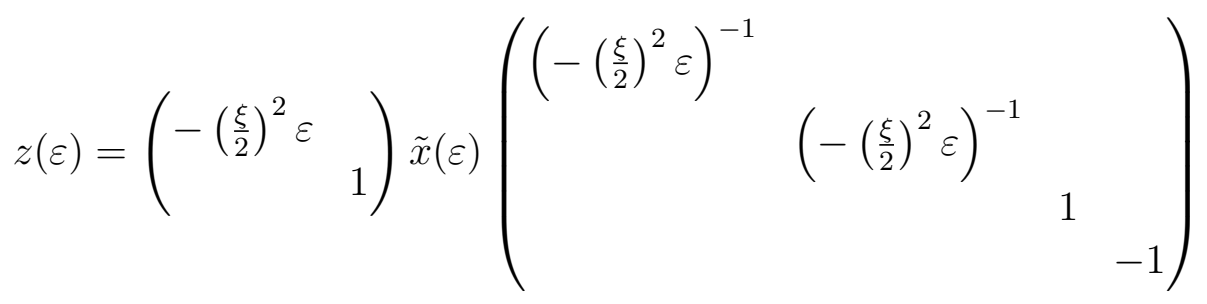

with $\tilde{x}(\varepsilon)=\left(\begin{array}{cccc}1 & 0 & 0 & 1 \\ 0 & x & 1 & -1\end{array}\right)$ and $x=-\left(\frac{\xi}{2}\right)^{2} \varepsilon$, we see that the GHG functions $\Phi(\tilde{\alpha} ; z(\varepsilon))$ and $\Phi(\tilde{\alpha} ; \tilde{x}(\varepsilon))$ are related as $\Phi(\tilde{\alpha} ; z(\varepsilon))=-x^{c-1} \Phi(\tilde{\alpha} ; \tilde{x}(\varepsilon))$ by virtue of the formula (8.1). Therefore we make a change of unknowns $u \mapsto v$ by $u=x^{-c+1} v$ to $(\mathrm{K})$ to get

$$
\left[\left(\vartheta_{x}-x\right)\left(\vartheta_{x}-c+1\right)-a x\right] v=0
$$

which corresponds to $\Phi(\alpha(\varepsilon) ; z(\varepsilon))$ after the changes of variable $x=-\left(\frac{\xi}{2}\right)^{2} \varepsilon$ and of parameters $\tilde{\alpha}=\tilde{\alpha}(\varepsilon)$ Thus if we make a change of variable and parameters $x=-\left(\frac{\xi}{2}\right)^{2} \varepsilon$ and $\tilde{\alpha}=\tilde{\alpha}(\varepsilon)$ for (8.2) and take a limit $\varepsilon \rightarrow 0$, then we get the equation (B'). Summarizing the discussion above, we have the following.

Proposition 8.1. For the equation (K), we make a change of variables and parameters

$$
\begin{array}{ll}
x=-\left(\frac{\xi}{2}\right)^{2} \varepsilon, & u=\left(-\left(\frac{\xi}{2}\right)^{2} \varepsilon\right)^{\nu} v \\
a=-\nu+\varepsilon^{-1}, & c=-\nu+1 .
\end{array}
$$

Then the limit $\varepsilon \rightarrow 0$ gives the confluence $(K) \rightarrow\left(B^{\prime}\right)$.

8.3. Kummer $\rightarrow$ Hermite-Weber. To realize this limit process, it is needed to recover a parameter in $(\mathrm{K})$, which became invisible in the course of reduction from GHG system to Kummer's differential equation. In order to recover it, we make a change of independent variable $x \mapsto x^{\prime}$ by $x=\alpha_{1} x^{\prime}$ to $(\mathrm{K})$. Writing $x^{\prime}$ as $x$ again, we get the equation

$$
u^{\prime \prime}+\left(c-\alpha_{1} x\right) u^{\prime}-a \alpha_{1} u=0 .
$$

Since the solutions of (8.3) are given by the integral

$$
u=\int e^{\alpha_{1} x t}(1-t)^{c-a-1} t^{a-1} d t
$$


it is seen that the equation (8.3) corresponds to the GHG system of type $(2,1,1)$ with

$$
\tilde{x}=\left(\begin{array}{cccc}
1 & 0 & 1 & 0 \\
0 & x & -1 & 1
\end{array}\right), \quad \tilde{\alpha}=\left(-c, \alpha_{1}, c-a-1, a-1\right) .
$$

Note that the Hermite-Weber equation (H-W') corresponds to the GHG system of type $(3,1)$ with

$$
w=\left(\begin{array}{cccc}
1 & 0 & 0 & 0 \\
0 & 1 & \xi & 1
\end{array}\right), \quad \alpha=(\nu-1,0,1,-\nu-1)
$$

Then the recipe of the confluence described in Theorem 5.3 guides us to consider the GHG system of type $(2,1,1)$ with

$$
\begin{aligned}
& z(\varepsilon)=w g(\varepsilon)=\left(\begin{array}{cccc}
1 & 0 & 1 & 0 \\
0 & 1 & \varepsilon+\varepsilon^{2} \xi & 1
\end{array}\right), \quad g(\varepsilon)=\left(\begin{array}{cccc}
1 & & 1 & \\
& 1 & \varepsilon & \\
& \varepsilon^{2} & \\
& & 1
\end{array}\right), \\
& \alpha(\varepsilon)=\alpha^{t} g(\varepsilon)^{-1}=\left(\nu-1-\varepsilon^{-2},-\varepsilon^{-1}, \varepsilon^{-2},-\nu-1\right) .
\end{aligned}
$$

Noting

$$
z(\varepsilon)=\left(\begin{array}{cc}
1 & \\
& -\left(\varepsilon+\varepsilon^{2} \xi\right)
\end{array}\right) \tilde{x}(\varepsilon)\left(\begin{array}{cccc}
1 & & & \\
& 1 & & \\
& & 1 & \\
& & & -\left(\varepsilon+\varepsilon^{2} \xi\right)^{-1}
\end{array}\right)
$$

with

$$
\tilde{x}(\varepsilon)=\left(\begin{array}{cccc}
1 & 0 & 1 & 0 \\
0 & x & -1 & 1
\end{array}\right), \quad x=-\left(\varepsilon+\varepsilon^{2} \xi\right)^{-1},
$$

we see that the GHG functions $\Phi(\tilde{\alpha} ; z(\varepsilon))$ and $\Phi(\tilde{\alpha} ; \tilde{x}(\varepsilon))$ of type $(2,1,1)$ are related as $\Phi(\tilde{\alpha} ; z(\varepsilon))=x^{a} \Phi(\tilde{\alpha} ; \tilde{x}(\varepsilon))$ by virtue of the formula (8.1). Hence we make a change of unknown $u \mapsto v$ defined by $u=x^{-a} v$ to the equation (8.3) and get

$$
\left[\left(\vartheta-a+c-1-\alpha_{1} x\right)(\vartheta-a)-a \alpha_{1} x\right] v=0 .
$$

We can check that, if we make a change of variable $x \mapsto \xi$ defined by (8.7) and a change of paramters

$$
a=-\nu, \quad \alpha_{1}=-\varepsilon^{-1}, \quad c=\varepsilon^{-2}-\nu+1
$$

induced from (8.6), then we get the equation (H-W') in the limit $\varepsilon \rightarrow 0$. We summarize the above process as follows.

Proposition 8.2. The change of variables

$$
x=\varepsilon^{-1}\left(\varepsilon+\varepsilon^{2} \xi\right)^{-1}, \quad u=\left(-\varepsilon-\varepsilon^{2} \xi\right)^{-\nu} v
$$

and the change of paramters given by (8.9) for the the Kummer's equation (K) induces the confluence to the Hermite-Weber equation $\left(H-W^{\prime}\right)$ as $\varepsilon \rightarrow 0$. 
8.4. Bessel $\rightarrow$ Airy. To apply the framework of confluence for GHG system to this case, we must recover a parameter in the equation (B') which disappeared in the course of reduction of GHG system of type $(2,2)$ to the equation (B'). Explicitly, we introduce a parameter $\alpha_{1}$ in (B') so that we can treat the integrals

$$
\int e^{\alpha_{1} t-\alpha_{1} \frac{x^{2}}{4 t}} t^{-\nu-1} d t
$$

instead of those for (B'). To derive the differential equation for (8.10) from (B'), we make a change of variable $x \mapsto x^{\prime}=x / \alpha_{1}$. Writing again $x^{\prime}$ as $x$, we get

$$
\left[\left(\vartheta_{x}+\nu\right)^{2}+\alpha_{1}^{2} x^{2}-\nu^{2}\right] u=0 .
$$

Viewing the integral representation (8.10) for (B"), we see that (B") corresponds to the GHG system with

$$
\tilde{x}=\left(\begin{array}{cccc}
1 & 0 & 0 & \frac{x^{2}}{4} \\
0 & 1 & 1 & 0
\end{array}\right), \quad \tilde{\alpha}=\left(-\nu_{1}-1, \alpha_{1}, \nu-1,-\alpha_{1}\right) .
$$

Let $w$ and $\alpha$ be the data for the GHG system corresponding to (A). Then the recipe of the confluence is to consider the GHG system of type $(2,2)$ with

$$
\begin{aligned}
& z(\varepsilon)=w g(\varepsilon)=\left(\begin{array}{cccc}
1 & 0 & 1 & 0 \\
0 & 1 & \varepsilon-\varepsilon^{3} \xi & 1-3 \varepsilon^{2} \xi
\end{array}\right), \quad g(\varepsilon)=\left(\begin{array}{cccc}
1 & & 1 & \\
& 1 & \varepsilon & 1 \\
& \varepsilon^{2} & 2 \varepsilon \\
& \varepsilon^{3} & 3 \varepsilon^{2}
\end{array}\right), \\
& \alpha(\varepsilon)=\alpha^{t} g(\varepsilon)^{-1}=\left(-2-2 \varepsilon^{-3}, e^{-2}, 2 \varepsilon^{-3},-\varepsilon^{-2}\right) .
\end{aligned}
$$

To relate this GHG system to the equation (B"), we decompose $z(\varepsilon)$ as

$$
z(\varepsilon)=\left(\begin{array}{cc}
1 & X(\varepsilon) \\
& 1
\end{array}\right) \tilde{x}(\varepsilon)\left(\begin{array}{cccc}
1 & X(\varepsilon) & & \\
& 1 & & \\
& & X(\varepsilon) & X^{\prime}(\varepsilon) \\
& & & X(\varepsilon)
\end{array}\right) \quad, \quad \tilde{x}(\varepsilon)=\left(\begin{array}{cccc}
1 & 0 & 0 & X^{\prime}(\varepsilon) \\
0 & 1 & 1 & 0
\end{array}\right)
$$

where

$$
X(\varepsilon)=\left(\varepsilon-\varepsilon^{3} \xi\right)^{-1}, \quad X^{\prime}(\varepsilon)=\frac{d X(\varepsilon)}{d \varepsilon}=-\frac{1-3 \varepsilon^{2} \xi}{\left(\varepsilon-\varepsilon^{3} \xi\right)^{-2}} .
$$

Then we see that the GHG functions $\Phi(\tilde{\alpha} ; z(\varepsilon))$ and $\Phi(\tilde{\alpha} ; \tilde{x}(\varepsilon))$ are related as

$$
\Phi(\tilde{\alpha} ; \tilde{x}(\varepsilon))=X(\varepsilon)^{-\nu-1} e^{\alpha_{1}\left(X(\varepsilon)-X^{\prime}(\varepsilon)\right)} \Phi(\tilde{\alpha} ; z(\varepsilon))
$$

by virtue of the formula (8.1). So if we make a change of variable, unkown and parameters

$$
\begin{aligned}
\frac{x^{2}}{4} & =X^{\prime}(\varepsilon)=-\left(1-3 \varepsilon^{2} \xi\right)\left(\varepsilon-\varepsilon^{3} \xi\right)^{-2} \\
u & =X(\varepsilon)^{-2 \varepsilon^{-3}} e^{\varepsilon^{-2}\left(X(\varepsilon)-X^{\prime}(\varepsilon)\right)} v \\
\nu & =-1-2 \varepsilon^{-3}, \quad \alpha_{1}=\varepsilon^{-2}
\end{aligned}
$$

to (B"), then we get the Airy equation (A) by letting $\varepsilon \rightarrow 0$. 
8.5. Hermite-Weber $\rightarrow$ Airy. To derive the $(\mathrm{A})$ from (H-W') by confluence, we must recover parameters in (H-W') which disappeared in the course of reduction from the GHG system of type $(3,1)$ to $\left(H_{-}-W^{\prime}\right)$. To this end we recall that the solutions of $\left(\mathrm{H}-\mathrm{W}^{\prime}\right)$ has the integral representation

$$
u(x)=\int e^{x t-\frac{1}{2} t^{2}} t^{-\nu-1} d t,
$$

which is the GHG function of type $(3,1)$ corresponding to

$$
\tilde{x}=\left(\begin{array}{cccc}
1 & 0 & 0 & 0 \\
0 & 1 & x & 1
\end{array}\right), \quad \tilde{\alpha}=\left(\alpha_{0}, \alpha_{1}, \alpha_{2}, \alpha_{3}\right)=(\nu-1,0,1,-\nu-1) .
$$

We recover the parameters $\alpha_{1}, \alpha_{2}$ so that we can treat

$$
v(x)=\int e^{\alpha_{1} t+\alpha_{2}\left(x t-\frac{1}{2} t^{2}\right)} t^{-\nu-1} d t
$$

instead of (8.12). To derive the equation for $v(x)$ from (H-W'), we make a change of independent variable $x \mapsto x^{\prime}$ :

$$
x=\alpha_{1} \alpha_{2}^{-\frac{1}{2}}+\alpha_{2}^{\frac{1}{2}} x^{\prime}
$$

to $(\mathrm{H}-\mathrm{W})$ and write again $x^{\prime}$ as $x$, we get

$$
u^{\prime \prime}-\left(\alpha_{1}+\alpha_{2} x\right) u^{\prime}+\nu \alpha_{2} u=0 .
$$

On the other hand, the Airy equation (A) corresponds to the GHG system of type (4) with

$$
w=\left(\begin{array}{cccc}
1 & 0 & 0 & 0 \\
0 & 1 & 0 & -\xi
\end{array}\right), \quad \alpha=(-2,0,0,-1) .
$$

Then the recipe of the confluence in Theorem 5.3 tells us to consider the GHG system of type $(3,1)$ with

$$
\begin{aligned}
& z(\varepsilon)=w g(\varepsilon)=\left(\begin{array}{cccc}
1 & 0 & 0 & 1 \\
0 & 1 & 0 & \varepsilon-\varepsilon^{3} \xi
\end{array}\right), \quad g(\varepsilon)=\left(\begin{array}{cccc}
1 & & & 1 \\
& 1 & & \varepsilon \\
& 1 & \varepsilon^{2} \\
& & \varepsilon^{3}
\end{array}\right), \\
& \alpha(\varepsilon)=\alpha^{t} g(\varepsilon)^{-1}=\left(-2+\varepsilon^{-3}, \varepsilon^{-2}, \varepsilon^{-1},-\varepsilon^{-3}\right) .
\end{aligned}
$$

To relate the GHG system to the equation (8.15), we decompose $z(\varepsilon)$ as

$$
z(\varepsilon)=\left(\begin{array}{cc}
1 & x \\
& 1
\end{array}\right) \tilde{x}(\varepsilon) h, \quad h=\left(\begin{array}{cccc}
1 & x & x^{2} & \\
& 1 & x & \\
& & 1 & \\
& & & x
\end{array}\right), \quad x=\left(\varepsilon-\varepsilon^{3} \xi\right)^{-1}
$$

where $\tilde{x}(\varepsilon)$ is the matrix obtained from $\tilde{x}$ in (8.13) by the substitution $x=\left(\varepsilon-\varepsilon^{3} \xi\right)^{-1}$. By virtue of the formula (8.1), the GHG functions of type $(3,1)$ for $z(\varepsilon)$ and $\tilde{x}(\varepsilon)$ are related as

$$
\Phi(\tilde{\alpha} ; z(\varepsilon))=\chi_{(3,1)}\left(\tilde{\alpha} ; h^{-1}\right) \Phi(\tilde{\alpha} ; \tilde{x}(\varepsilon))=e^{-\alpha_{1} x-\frac{1}{2} \alpha_{2} x^{2}} x^{-\alpha_{3}} \Phi(\tilde{\alpha} ; \tilde{x}(\varepsilon))
$$


Thus we get the change of unknown $u \mapsto v: u=e^{\alpha_{1} x+\frac{1}{2} \alpha_{2} x^{2}} x^{\alpha_{3}} v$ together with $x=$ $\left(\varepsilon-\varepsilon^{3} \xi\right)^{-1}$ and $\tilde{\alpha}=\alpha(\varepsilon)$ to get the differential equation for the GHG function $\Phi(\alpha(\varepsilon) ; \tilde{z})$.

To summarize, we have the following.

Proposition 8.3. The confluence $\left(H^{\prime}\right) \rightarrow(A)$ is carried out by the following steps:

(1) the change of independent variable $x \mapsto x^{\prime}$ defined by (8.14) and that of unknown $u \mapsto v$ defined by

$$
u=e^{\alpha_{1} x^{\prime}+\frac{1}{2} \alpha_{2} x^{\prime 2}} x^{\prime \alpha_{3}} v
$$

(2) the second change of independent variable and that of parameters:

$$
x^{\prime}=\left(\varepsilon-\varepsilon^{3} \xi\right)^{-1}, \quad \alpha_{1}=\varepsilon^{-2}, \quad \alpha_{2}=\varepsilon^{-1}, \quad \nu=-1+\varepsilon^{-3} .
$$

(3) to take a limit $\varepsilon \rightarrow 0$ in the equation obtained by the steps (1) and (2).

8.6. Appell's $F_{1}$ and its confluent family. Appell's hypergeometric function $F_{1}$ and its classically known confluent family $([2])$ are defined by the power series:

$$
\left\{\begin{aligned}
F_{1}\left(a, b, b^{\prime}, c ; x, y\right) & =\sum_{m, n=0}^{\infty} \frac{(a)_{m+n}(b)_{m}\left(b^{\prime}\right)_{n}}{(c)_{m+n} m ! n !} x^{m} y^{n} \\
\Phi_{1}(a, b, c ; x, y) & =\sum_{m, n=0}^{\infty} \frac{(a)_{m+n}(b)_{m}}{(c)_{m+n} m ! n !} x^{m} y^{n}=\lim _{\varepsilon \rightarrow 0} F_{1}(a, b,-1 / \varepsilon, c ; x,-\varepsilon y), \\
\Phi_{2}\left(b, b^{\prime}, c ; x, y\right) & =\sum_{m, n=0}^{\infty} \frac{(b)_{m}\left(b^{\prime}\right)_{n}}{(c)_{m+n} m ! n !} x^{m} y^{n}=\lim _{\varepsilon \rightarrow 0} F_{1}\left(-1 / \varepsilon, b, b^{\prime}, c ;-\varepsilon x,-\varepsilon y\right), \\
\Phi_{3}(b, c ; x, y) & =\sum_{m, n=0}^{\infty} \frac{(b)_{m}}{(c)_{m+n} m ! n !} x^{m} y^{n}=\lim _{\varepsilon \rightarrow 0} \Phi_{2}(b,-1 / \varepsilon, c ; x,-\varepsilon y),
\end{aligned}\right.
$$

where $(a)_{m}=\Gamma(a+m) / \Gamma(a)$ is the Pochhammer's symbol. The functions $F_{1}, \Phi_{1}, \Phi_{2}$ and $\Phi_{3}$ satisfy the systems of partial differential equations:

$$
\begin{aligned}
& \left\{\begin{array}{l}
x(1-x) u_{x x}+y(1-x) u_{x y}+\{c-(a+b+1) x\} u_{x}-b y u_{y}-a b u=0, \\
y(1-y) u_{y y}+x(1-y) u_{x y}+\left\{c-\left(a+b^{\prime}+1\right) y\right\} u_{y}-b^{\prime} x u_{x}-a b^{\prime} u=0,
\end{array}\right. \\
& \left\{\begin{array}{l}
x(1-x) u_{x x}+y(1-x) u_{x y}+\{c-(a+b+1) x\} u_{x}-b y u_{y}-a b u=0, \\
y u_{y y}+x u_{x y}+(c-y) u_{y}-x u_{x}-a u=0,
\end{array}\right. \\
& \left\{\begin{array}{l}
x u_{x x}+y u_{x y}+(c-x) u_{x}-b u=0, \\
y u_{y y}+x u_{x y}+(c-y) u_{y}-b^{\prime} u=0,
\end{array}\right. \\
& \left\{\begin{array}{l}
x u_{x x}+y u_{x y}+(c-x) u_{x}-b u=0, \\
y u_{y y}+x u_{x y}+c u_{y}-u=0,
\end{array}\right.
\end{aligned}
$$

respectively, where the suffix $x$ or $y$ in $u_{x}$ or $u_{y}$ denotes the derivation with respect to $x$ or $y$. It is known that the limit processes in (8.16) induce the confluence of the systems $\left(F_{1}\right) \rightarrow\left(\Phi_{1}\right),\left(F_{1}\right) \rightarrow\left(\Phi_{2}\right)$ and $\left(\Phi_{1}\right) \rightarrow\left(\Phi_{2}\right)$. We shall explain that these confluence processes can be treated in a unified way by Theorem 5.3. To relate the systems $\left(F_{1}\right)$, $\left(\Phi_{1}\right),\left(\Phi_{2}\right)$ and $\left(\Phi_{3}\right)$ to the GHG systems with the Young diagrams of weight 5 , we recall 
the integral representations for the solutions, in which we omit the domains of integration since we don't need them:

$$
\begin{aligned}
F_{1} & =C_{1} \int(1-y t)^{-b^{\prime}} t^{a-1}(1-t)^{c-a-1}(1-x t)^{-b} d t \\
& =C_{2} \int(1-x s-y t)^{-a} s^{b-1} t^{b^{\prime}-1}(1-s-t)^{c-b-b^{\prime}-1} d s d t \\
\Phi_{1} & =C_{3} \int e^{y t} t^{a-1}(1-t)^{c-a-1}(1-x t)^{-b} d t \\
\Phi_{2} & =C_{4} \int e^{-t} t^{b+b^{\prime}-c}(y+t)^{-b^{\prime}}(x+t)^{-b} d t \\
& =C_{5} \int e^{x s+y t} s^{b-1} t^{b^{\prime}-1}(1-s-t)^{c-b-b^{\prime}-1} d s d t, \\
\Phi_{3} & =C_{6} \int e^{-t-\frac{y}{t}} t^{b-c}(x+t)^{-b} d t,
\end{aligned}
$$

where $C_{k}$ are some constants depending on the parameters $a, b, b^{\prime}$ and $c$. From these 1dimensional integral representations, we see that $\left(F_{1}\right),\left(\Phi_{1}\right),\left(\Phi_{2}\right)$ and $\left(\Phi_{3}\right)$ are the GHG systems of type $(1,1,1,1,1),(2,1,1,1),(2,1,1,1)$ and $(2,2,1)$ on the space of matrices:

$$
\begin{aligned}
X_{(1,1,1,1,1)} & =\left\{\left(\begin{array}{ccccc}
1 & 1 & 0 & 1 & 1 \\
0 & -y & 1 & -1 & -x
\end{array}\right) \mid x y(x-1)(y-1)(x-y) \neq 0\right\}, \\
X_{(2,1,1,1)} & =\left\{\left(\begin{array}{ccccc}
1 & 0 & 0 & 1 & 1 \\
0 & y & 1 & -1 & -x
\end{array}\right) \mid x y(x-1) \neq 0\right\}, \\
X_{(2,1,1,1)}^{\prime} & =\left\{\left(\begin{array}{ccccc}
1 & 0 & 0 & y & x \\
0 & 1 & 1 & 1 & 1
\end{array}\right) \mid x y(x-y) \neq 0\right\}, \\
X_{(2,2,1)} & =\left\{\left(\begin{array}{lllll}
1 & 0 & 0 & y & x \\
0 & 1 & 1 & 0 & 1
\end{array}\right) \mid x y \neq 0\right\},
\end{aligned}
$$

respectively, with parameters $\alpha_{\lambda}$ :

$$
\begin{aligned}
\alpha_{(1,1,1,1,1)} & =\left(b+b^{\prime}-c,-b^{\prime}, a-1, c-a-1,-b\right), \\
\alpha_{(2,1,1,1)} & =(b-c, 1, a-1,-b,-b), \\
\alpha_{(2,1,1,1)}^{\prime} & =\left(c-2,-1, b+b^{\prime}-c,-b,-b^{\prime}\right), \\
\alpha_{(2,2,1)} & =(c-2,-1, b-c,-1,-b) .
\end{aligned}
$$

Corresponding to 2-dimensional integral representations for $F_{1}$ and $\Phi_{2}$, we can regard $\left(F_{1}\right)$ and $\left(\Phi_{2}\right)$ as the GHG systems on the matrix spaces

$$
\begin{aligned}
Y_{(1,1,1,1,1)} & =\left\{\left(\begin{array}{ccccc}
1 & 1 & 0 & 0 & 1 \\
0 & -x & 1 & 0 & -1 \\
0 & -y & 0 & 1 & -1
\end{array}\right) \mid x y(x-1)(y-1)(x-y) \neq 0\right\}, \\
Y_{(2,1,1,1)} & =\left\{\left(\begin{array}{ccccc}
1 & 0 & 0 & 0 & 1 \\
0 & x & 1 & 0 & -1 \\
0 & y & 0 & 1 & -1
\end{array}\right) \mid x y(x-y) \neq 0\right\}
\end{aligned}
$$


with the parameters:

$$
\begin{aligned}
\alpha_{(1,1,1,1,1)} & =\left(a-c,-a, b-1, b^{\prime}-1, c-b-b^{\prime}-1\right), \\
\alpha_{(2,1,1,1)} & =\left(-c, 1, b-1, b^{\prime}-1, c-b-b^{\prime}-1\right),
\end{aligned}
$$

respectively.

8.7. Confluence $\left(F_{1}\right) \rightarrow\left(\Phi_{1}\right)$. In the present and following subsections, we denote by $x, y$ the independent variables for the system of source (namely $\left(F_{1}\right)$ in the present case) and by $\xi, \eta$ the independent variables for the system of target (namely $\left(\Phi_{1}\right)$ in the present case) in the process of confluence. We also denote by $z$ (resp. $w$ ) and $\tilde{\alpha}$ (resp. $\alpha$ ) the matrix variable and parameters for the GHG system corresponding to the source (resp. target) system. In the case $\left(F_{1}\right) \rightarrow\left(\Phi_{1}\right)$,

$$
z=\left(\begin{array}{ccccc}
1 & 1 & 0 & 1 & 1 \\
0 & -y & 1 & -1 & -x
\end{array}\right), \quad \tilde{\alpha}=\left(b+b^{\prime}-c,-b^{\prime}, a-1, c-a-1,-b\right)
$$

and

$$
w=\left(\begin{array}{ccccc}
1 & 0 & 0 & 1 & 1 \\
0 & \eta & 1 & -1 & -\xi
\end{array}\right), \quad \alpha=(b-c, 1, a-1, c-a-1,-b) .
$$

Then the recipe of the confluence given in Theorem 5.3 is to consider the GHG system of type $(1,1,1,1,1)$ with

$$
\begin{gathered}
z(\varepsilon)=w g(\varepsilon)=\left(\begin{array}{ccccc}
1 & 1 & 0 & 1 & 1 \\
0 & \varepsilon \eta & 1 & -1 & -\xi
\end{array}\right), \quad g(\varepsilon)=\left(\begin{array}{ccccc}
1 & 1 & & & \\
& \varepsilon & & & \\
& 1 & & \\
& & 1 & \\
& & & 1
\end{array}\right), \\
\alpha(\varepsilon)=\alpha^{t} g(\varepsilon)^{-1}=\left(b-c-\varepsilon^{-1}, \varepsilon^{-1}, a-1, c-a-1,-b\right) .
\end{gathered}
$$

Comparing these with (8.19), we get the change of variables and parameters:

$$
x=\xi, \quad y=-\varepsilon \eta, \quad b^{\prime}=-\varepsilon^{-1}
$$

for the system $\left(F_{1}\right)$, which induces the confluence $\left(F_{1}\right) \rightarrow\left(\Phi_{1}\right)$ in the limit $\varepsilon \rightarrow 0$.

8.8. Confluence $\left(F_{1}\right) \rightarrow\left(\Phi_{2}\right)$. In this case, it is convenient to use the GHG systems on the matrix spaces $Y_{(1,1,1,1,1)}$ and $Y_{(2,1,1,1)}$. Let

$$
z=\left(\begin{array}{ccccc}
1 & 1 & 0 & 0 & 1 \\
0 & -x & 1 & 0 & -1 \\
0 & -y & 0 & 1 & -1
\end{array}\right), \quad \tilde{\alpha}=\left(a-c,-a, b-1, b^{\prime}-1, c-b-b^{\prime}-1\right)
$$

and

$$
w=\left(\begin{array}{ccccc}
1 & 0 & 0 & 0 & 1 \\
0 & \xi & 1 & 0 & -1 \\
0 & \eta & 0 & 1 & -1
\end{array}\right), \quad \alpha=\left(-c, 1, b-1, b^{\prime}-1, c-b-b^{\prime}-1\right)
$$


be the matrices and parameters for the systems $\left(F_{1}\right)$ and $\left(\Phi_{2}\right)$, respectively. Then we apply the recipe of Theorem 5.3: put

$$
\begin{aligned}
& z(\varepsilon)=w g(\varepsilon)=\left(\begin{array}{ccccc}
1 & 0 & 0 & 0 & 1 \\
0 & \varepsilon \xi & 1 & 0 & -1 \\
0 & \varepsilon \eta & 0 & 1 & -1
\end{array}\right), \quad g(\varepsilon)=\left(\begin{array}{ccccc}
1 & 1 & & \\
& \varepsilon & & \\
& 1 & & \\
& & 1 & \\
& & & 1
\end{array}\right) \\
& \alpha(\varepsilon)=\alpha^{t} g(\varepsilon)^{-1}=\left(-c-\varepsilon^{-1}, \varepsilon^{-1}, b-1, b^{\prime}-1, c-b-b^{\prime}-1\right)
\end{aligned}
$$

and consider the GHG system of type $(1,1,1,1,1)$ with $z(\varepsilon)$ and $\alpha(\varepsilon)$. Comparing $z(\varepsilon)$ and $\alpha(\varepsilon)$ with $z$ and $\alpha$ in (8.21), we find the desired change of variables and parameters:

$$
x=-\varepsilon \xi, \quad y=-\varepsilon \eta, \quad a=-\varepsilon^{-1}
$$

for the system $\left(F_{1}\right)$, which induces the confluence $\left(F_{1}\right) \rightarrow\left(\Phi_{2}\right)$ in the limit $\varepsilon \rightarrow 0$.

8.9. Confluence $\left(\Phi_{2}\right) \rightarrow\left(\Phi_{3}\right)$. Let $z \in X_{(2,1,1,1)}^{\prime}$ and $\tilde{\alpha}$ be the matrix and parameters for $\left(\Phi_{2}\right)$ and let $w \in X_{(2,2,1)}$ and $\alpha$ be those for $\left(\Phi_{3}\right)$ given in (8.17) and (8.18). Then, as in the previous case, we consider the GHG system of type $(2,1,1,1)$ with

$$
\begin{aligned}
& z(\varepsilon)=w g(\varepsilon)=\left(\begin{array}{ccccc}
1 & 0 & 0 & \varepsilon \eta & \xi \\
0 & 1 & 1 & 1 & 1
\end{array}\right), \quad g(\varepsilon)=\left(\begin{array}{ccccc}
1 & & & & \\
& 1 & & & \\
& 1 & 1 & \\
& & \varepsilon & \\
& & & 1
\end{array}\right), \\
& \alpha(\varepsilon)=\alpha^{t} g(\varepsilon)^{-1}=\left(c-2,-1, b-c+\varepsilon^{-1},-\varepsilon^{-1},-b\right) .
\end{aligned}
$$

Then putting $z=z(\varepsilon)$ and $\tilde{\alpha}=\alpha(\varepsilon)$, we have the change of variables and parameters

$$
x=\xi, \quad y=\varepsilon \eta, \quad b^{\prime}=\varepsilon^{-1},
$$

which induces the confluence $\left(\Phi_{2}\right) \rightarrow\left(\Phi_{3}\right)$ when $\varepsilon \rightarrow 0$.

\section{REFERENCES}

[1] K. Aomoto, Les équations aux différences linéaires et les intégrales des fonctions multiformes, J. Fac. Sci. Univ. Tokyo, Sect. IA Math. 22 (1975), 271-297.

[2] P. Appell et J. Kampé de Fériet, Fonctions hypergeometriques et hypersphériques, Gauthier-Villars, 1926.

[3] V. Arnold, A. Varchenko and S. Goussein-Zadé, Singularité des applications différentiables, Vol. 1,2, Mir Moscou, 1986 .

[4] Erdélyi et al., Higher transcendental functions, Vol. 1 MacGraw-Hill Book company, Inc., New YorkToronto-London, 1953.

[5] M. Fujiwara, Algebra (in Japanese), Vol. 1, Uchida Rokakuho Publishing Co., Ltd, 1966.

[6] I. M. Gelfand, S.G. Gindikin and M.I. Graev, Integral geometry in affine and projective spaces, J. Soviet Math. 18 (1982), 39-167.

[7] I. M. Gelfand, General theory of hypergeometric functions, Dokl. Akad. Nauk. SSSR 288 (1986), 14-18, English translation, Soviet Math. Dokl. 33 (1986), 9-13.

[8] I. M. Gelfand and S. I. Gelfand, Generalized hypergeometric equations, Dokl. Akad. Nauk. SSSR 288 (1986), 279-283, English transl., Soviet Math. Dokl. 33 (1986), 643-646. 
[9] I. M. Gelfand, V. S. Retahk and V. V. Serganova, Generalized Airy funcitons, Schubert cells, and Jordan groups, Dokl. Akad. Nauk. SSSR 298 (1988), 17-21, English transl., Soviet Math. Dokl. 37 (1988), 8-12.

[10] K. Iwasaki, H. Kimura, S. Shimomura and M. Yoshida, From Gauss to Painlevé, Aspects Math. E16, Friedr, Vieweg \& Sohn, Braunschweig, 1991.

[11] T. Inui, Special functions (in Japanese), Iwanami Shoten, 1962.

[12] F. John, The Ultrahyperbolic differential equation with four independent variables, Duke Math. J. 4 (1938), 300-322.

[13] T. Kakehi, Range characterization of Radon transforms on complex projective spaces, J. Math. Kyoto Univ. 32 (1992), 387-399.

[14] H. Kimura, On rational de Rham cohomology associated with the generalized Airy functions, Ann. Scuola Norm. Sup. Pisa Cl. Sci. 24 (1997), 351-366.

[15] H. Kimura, On the homology group associated with the general Airy integral, Kumamoto J. Math. 10 (1997), 11-29.

[16] H. Kimura, Y. Haraoka and K. Takano, The generalized confluent hypergeometric functions, Proc. Japan Acad. Ser. A Math. Sci. 68 (1992), 290-295.

[17] H. Kimura, Y. Haraoka and K. Takano, On confluences of the general hypergeometric systems. Proc. Japan Acad. Ser. A Math. Sci. 69 (1993), 99-104.

[18] H. Kimura and T. Koitabashi, Normalizer of maximal abelian subgroups of $G L(n)$ and the general hypergeometric functions, Kumamoto J. Math. 9 (1996), 13-43.

[19] K. Okamoto and H. Kimura, On particular solutions of Garnier systems and the hypergeometric functions of several variables, Quart. J. Math. Oxford Ser. (2) 37 (1986), 61-80.

Department of Mathematics

KUMAMOTO UNIVERSITY

КUмАмото 860

JAPAN

E-mail address: hiro@sci.kumamoto-u.ac.jp

Department of Mathematics

KoBe UNIVERSITY

KoBe 657

JAPAN

E-mail address: takano@math.kobe-u.ac.jp 